Mon. Not. R. Astron. Soc. 000, 000-000 (0000) Printed 22 October $2018 \quad$ (MN LATEX style file v1.4)

\title{
The Diversity and Similarity of Simulated Cold Dark Matter Halos
}

\author{
Julio F. Navarro ${ }^{1,5}$, Aaron Ludlow ${ }^{1}$, Volker Springel ${ }^{2}$, Jie Wang ${ }^{2}$, \\ Mark Vogelsberger ${ }^{2}$, Simon D.M. White ${ }^{2}$, Adrian Jenkins ${ }^{3}$, Carlos S. Frenk ${ }^{3}$, and \\ Amina Helmi ${ }^{4}$ \\ ${ }^{1}$ Dept. of Physics and Astronomy, University of Victoria, Victoria, BC, V8P 5C2, Canada \\ ' 2 Max-Planck-Institut für Astrophysik, Karl-Schwarzschild-Straße 1, 85740 Garching bei München, Germany \\ ' ${ }^{3}$ Institute for Computational Cosmology, Dep. of Physics, Univ. of Durham, South Road, Durham DH1 3LE, UK \\ ${ }^{4}$ Kapteyn Astronomical Institute, Univ. of Groningen, P.O. Box 800, 9700 AV Groningen, The Netherlands \\ ${ }^{5}$ Department of Astronomy, University of Massachusetts, Amherst, MA 01003-9305, USA
}

22 October 2018

\begin{abstract}
We study the mass, velocity dispersion, and anisotropy profiles of $\Lambda$ CDM halos using a suite of N-body simulations of unprecedented numerical resolution. The Aquarius Project follows the formation of 6 different galaxy-sized halos simulated several times at varying numerical resolution, allowing numerical convergence to be assessed directly. The highest resolution simulation represents a single dark matter halo using 4.4 billion particles, of which 1.1 billion end up within the virial radius. Our analysis confirms a number of results claimed by earlier work, and clarifies a few issues where conflicting claims may be found in the recent literature. The mass profile of $\Lambda \mathrm{CDM}$ halos deviates slightly but systematically from the form proposed by Navarro, Frenk \& White. The spherically-averaged density profile becomes progressively shallower inwards and, at the innermost resolved radius, the logarithmic slope is $\gamma \equiv-\operatorname{dln} \rho / \operatorname{dln} r \lesssim 1$. Asymptotic inner slopes as steep as the recently claimed $\rho \propto r^{-1.2}$ are clearly ruled out. The radial dependence of $\gamma$ is well approximated by a power-law, $\gamma \propto r^{\alpha}$ (the Einasto profile). The shape parameter, $\alpha$, varies slightly but significantly from halo to halo, implying that the mass profiles of $\Lambda \mathrm{CDM}$ halos are not strictly universal: different halos cannot, in general, be rescaled to look identical. Departures from similarity are also seen in velocity dispersion profiles and correlate with those in density profiles so as to preserve a power-law form for the spherically averaged pseudo-phase-space density, $\rho / \sigma^{3} \propto r^{-1.875}$. The index here is identical to that of Bertschinger's similarity solution for self-similar infall onto a point mass from an otherwise uniform Einstein-de Sitter Universe. The origin of this striking behaviour is unclear, but its robustness suggests that it reflects a fundamental structural property of $\Lambda$ CDM halos. Our conclusions are reliable down to radii below $0.4 \%$ of the virial radius, providing well-defined predictions for halo structure when baryonic effects are neglected, and thus an instructive theoretical template against which the modifications induced by the baryonic components of real galaxies can be judged.
\end{abstract}

Key words: cosmology: dark matter - methods: numerical

\section{INTRODUCTION}

A couple of decades of steady progress in the simulation of non-linear structures in a cold dark matter (CDM) dominated universe have resulted in significant advances in our

^ E-mail: jfn@uvic.ca understanding of the clustering of dark matter on the scale of galactic halos. There is now widespread consensus that the hierarchical assembly of CDM halos yields: (1) mass profiles that are approximately "universal" (i.e., independent of mass and cosmological parameters aside from simple physical scalings (Navarro et al., 1996, 1997, hereafter NFW), (2) strongly triaxial shapes, with a slight preference for nearly 
prolate systems (e.g., Frenk et al., 1988; Jing \& Suto, 2002; Allgood et al., 2006; Hayashi et al., 2007), (3) abundant, but non-dominant, substructure (Klypin et al., 1999; Moore et al., 1999a; Ghigna et al., 2000; Gao et al., 2004), and (4) "cuspy" inner mass profiles, where the central density increases systematically as the numerical resolution of the calculation is improved (see, e.g., NFW, Moore et al., 1999b; Fukushige \& Makino, 2001; Navarro et al., 2004; Diemand et al., 2005).

Despite this consensus, there are a number of issues where conflicting claims may be found in the recent literature, hindering the design and interpretation of observational tests aimed at validating or ruling out various aspects of the CDM theory on these scales. One contentious issue concerns the statistics, spatial distribution, and structure of substructure, and their consequences for the discovery and interpretation of possible signals of dark matter annihilation in the gamma-ray sky (Stoehr et al., 2003; Diemand et al., 2007; Kuhlen et al., 2008; Springel et al., 2008a, and references therein). The controversy extends to the structure of the inner cusps both of the main halo and of substructure halos, where some recent work has claimed a well-defined central slope of $\rho \propto r^{-1.2}$ (Diemand et al., 2004, 2005, 2008) whereas others have argued that no compelling evidence for such power-law behaviour is apparent (Navarro et al., 2004; Graham et al., 2006).

Considerable debate also surrounds whether the structure of CDM halos is truly "universal". This is indeed the case if halos have mass profiles that are well-described by two-parameter formulae, such as the NFW profile or some of its modifications; see, for example, Moore et al. (1999b, hereafter M99). These profiles have two scaling parameters (mass and size) but fixed shape, so that two different halos can, in principle, be rescaled to be indistinguishable from each other.

On the other hand, recent work suggests that at least three parameters may be needed to describe halo mass profiles accurately. An example is the Einasto formula (Einasto, 1965), shown by Navarro et al. (2004) to improve significantly the accuracy of the fits to the inner density profiles of simulated halos. It is unclear from that work, however, whether the improvement is due to the fact that the Einasto formula has a different asymptotic inner behaviour than NFW or to the extra shape parameter it introduces. Merritt et al. (2005, 2006) explored this further and argued that the third parameter is indeed needed to account faithfully for the curvature in the shape of the density profile. Merritt et al.'s conclusions have received support from the work of Gao et al. (2008) and Hayashi \& White (2008), who have stacked density profiles of many halos of similar mass to show that mean profile shape, and, in particular, the Einasto shape parameter $\alpha$ (see eq. 4 below), depends systematically on halo mass. This implies that the mass profile of $\Lambda \mathrm{CDM}$ halos is not strictly universal; no simple scaling of the average profile of cluster halos will provide an accurate fit to the average profile of galaxy halos.

Many of these controversies and uncertainties may be traced to the fact that earlier work has lacked the numerical resolution and the representative halo sample needed to settle the debate. For example, the dark matter annihilation flux observable from Earth depends crucially on resolving not only substructures but also the nested "substructure within substructure" expected from the hierarchical assembly of CDM halos. Only the most recent simulations have been able to begin addressing this issue (see, e.g., Diemand et al., 2008; Springel et al., 2008a,b).

A similar comment applies to the structure of the inner cusp, where pinning down the asymptotic inner behaviour of the dark matter density profile depends crucially on understanding the limitations introduced by, for example, finite particle number, gravitational softening, and time-stepping technique.

We have shown in earlier work (Power et al., 2003, hereafter P03) that, when suitable choices of the numerical parameters are made, the main factor determining the innermost radius where the mass profile may be measured reliably is the total number of particles used in the simulation. Empirically, the boundary of the region where numerical convergence is achieved roughly corresponds to the radius where the two-body relaxation time, $t_{\text {relax }}$, exceeds the age of the Universe. Since $t_{\text {relax }}$ scales roughly like the enclosed number of particles times the local orbital timescale, and the latter drops sharply toward the centre, extending the resolved region inwards even modestly requires a dramatic increase in the total number of particles.

These difficulties, coupled to the significant halo-to-halo scatter already seen in early work, imply that substantive progress on these issues requires a concerted numerical effort where several different halos are simulated with varying numerical resolution, so that cosmic variance and numerical convergence may be assessed directly.

These are the aims of The Aquarius Project, a recently completed suite of numerical simulations of the formation of galaxy-sized halos in the $\Lambda \mathrm{CDM}$ cosmogony. The series includes re-simulations of six different $\sim 10^{12} M_{\odot}$ halos where the number of particles is systematically varied. In one case, the same halo is simulated 5 times, increasing gradually the number of particles in the halo from about one million to $\sim 1.1$ billion within the virial radius. The highest resolution simulations of the other 5 halos have roughly 100-200 million particles each within the virialized region.

The simulation series has been presented recently by Springel et al. (2008a,b), where the interested reader may find relevant details. Our first paper (Springel et al., 2008a) deals with predictions of the annihilation signal whereas the second (Springel et al., 2008b) addresses the statistics, spatial distribution, and structure of dark matter substructures. Here we deal with the structure of the main halo, with special emphasis on the structure of the inner cusp. The plan of the paper is as follows. Sec. 2 summarizes briefly the numerical parameters of our simulations; Sec. 3 and 4 present our main results. We conclude with a brief discussion and summary in Sec. 5.

\section{THE NUMERICAL SIMULATIONS}

We present here for completeness a brief summary of the numerical simulations, and refer the reader to Springel et al. $(2008 \mathrm{a}, \mathrm{b})$ for further details. 

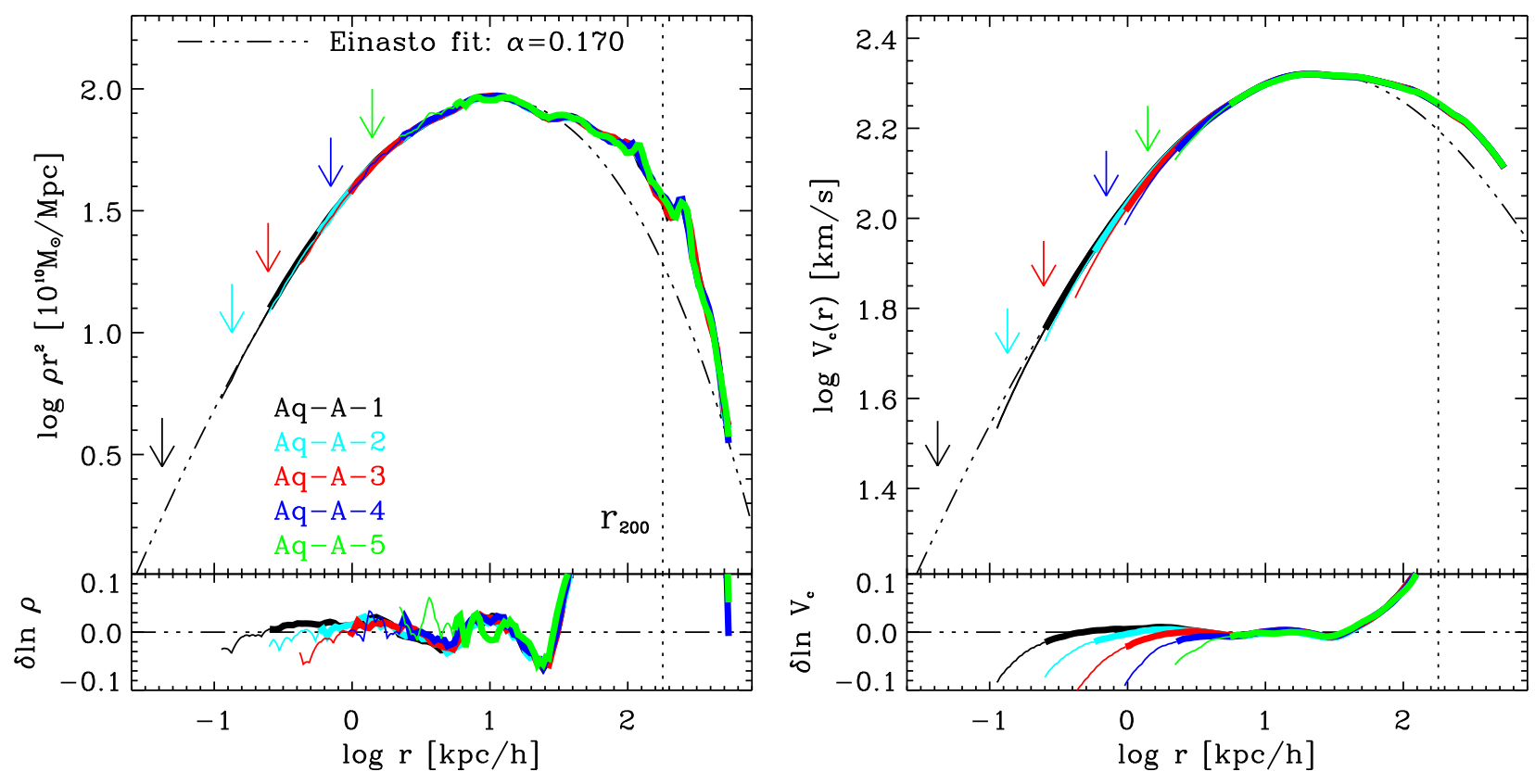

Figure 1. Spherically-averaged density (left) and circular velocity (right) profiles for the Aq-A halo simulation series. Different colours correspond to different resolution runs, as labeled in the figure. The density profile is multiplied by $r^{2}$ in order to emphasize small deviations. The bumps in the outer regions may be traced to the presence of substructure and unrelaxed tidal debris. Profiles are shown from $\sim 3 r_{200}$ down to the "convergence radius", $r_{\text {conv }}^{(1)}$, corresponding to the radius where the relaxation time, $t_{\text {relax }}$, is of the order of the age of the Universe. The thick portion of each profile indicates the region $r>r_{\text {conv }}^{(7)}$ where $t_{\text {relax }}$ is more than 7 times the age of the universe and where stricter convergence is achieved. Outside $r_{\text {conv }}^{(7)}$ circular velocity estimates converge to better than $2.5 \%$ (see Fig. 2). The dot-dashed line shows an Einasto profile with $\alpha=0.17$ matched at $\left(r_{-2}, \rho_{-2}\right)$, the peak in the $r^{2} \rho$ profile. This provides an excellent fit to the structure of the inner regions of the halo, as shown by the residuals plotted in the bottom panels. Arrows indicate the softening length $h_{s}$ of each simulation.

\subsection{The Cosmological Parameters}

All our simulations assume a $\Lambda$ CDM cosmogony with the following parameters: $\Omega_{\mathrm{m}}=0.25, \Omega_{\Lambda}=0.75, \sigma_{8}=0.9$, $n_{s}=1$, and Hubble constant $H_{0}=100 \mathrm{hm} \mathrm{s}^{-1} \mathrm{Mpc}^{-1}=$ $73 \mathrm{kms}^{-1} \mathrm{Mpc}^{-1}$. These cosmological parameters are the same adopted in previous numerical work by our group, such as the Millennium Simulation of Springel et al. (2005), and are consistent, within their uncertainties, with constraints derived from the WMAP 1- and 5-year data analyses (Spergel et al., 2003; Komatsu et al., 2008) and with the recent cluster abundance analysis of Henry et al. (2008).

\subsection{The Code}

The simulations were carried out with a new version of the GADGET (Springel et al., 2001; Springel, 2005) parallel cosmological code. This version, which we call GADGET-3, has been especially developed for this project, and implements a novel domain decomposition technique in order to achieve unprecedented dynamic range in massively-parallel computer systems without sacrificing load balancing or numerical accuracy. Time stepping is carried out with a kickdrift-kick leap-frog integrator where the timesteps are based on the local gravitational acceleration, together with a conservatively chosen maximum timestep for all particles.

Pairwise particle interactions are softened with a spline of scalelength $h_{s}$, so that they are strictly Newtonian for par- ticles separated by more than $h_{s}$. The resulting softening is roughly equivalent to a traditional Plummer-softening with scalelength $\epsilon_{G} \sim h_{s} / 2.8$. The gravitational softening length is kept fixed in comoving coordinates throughout the evolution of all our halos. The dynamics is then governed by a Hamiltonian and the phase-space density of the discretized particle system should be strictly conserved as a function of time (Springel, 2005).

\subsection{Halo Selection}

All halos in the Aquarius suite were identified for resimulation in a $900^{3}$-particle parent simulation of a $100 \mathrm{~h}^{-1} \mathrm{Mpc}$ box. The identification technique selects all $\sim 10^{12} M_{\odot}$ halos in the box and chooses, at random, a few of them that satisfy a mild isolation criterion (no neighbour exceeding half its mass within $1 h^{-1} \mathrm{Mpc}$ ). This criterion is only imposed in order to remove halos in the vicinity of massive groups and clusters, which may have evolved differently from the average.

Each halo is then resimulated at various resolutions, making sure that each resimulation shares the same power spectrum and phases at all resolved spatial frequencies. Initial displacements are imprinted using the Zeldovich approximation and a 'glass-like' uniform particle load (White, 1996). The $100 h^{-1} \mathrm{Mpc}$ simulation box is divided into a "high-resolution" region, which corresponds to the La- 


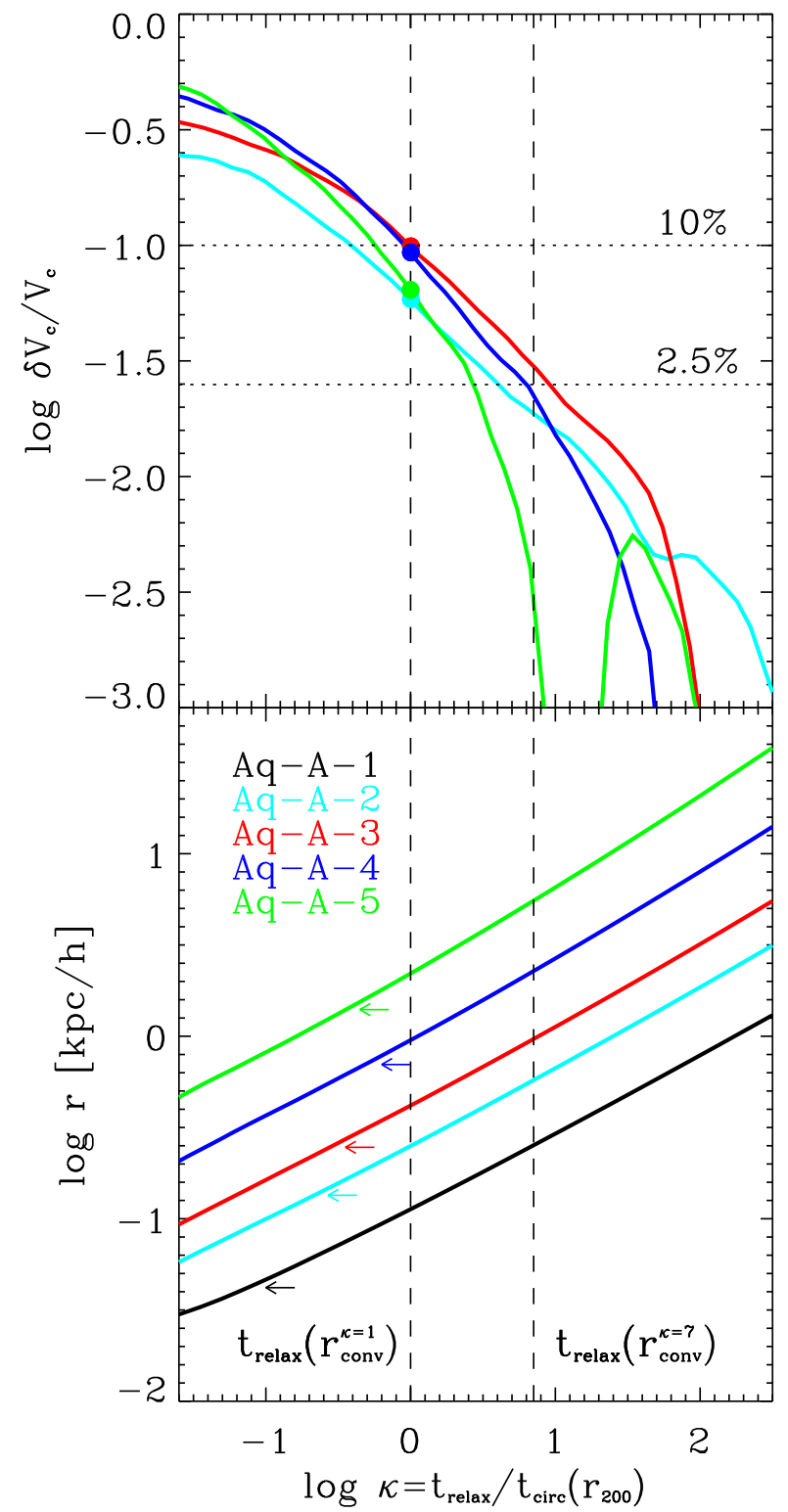

Figure 2. Top panel: Fractional deviations in the circular velocity profile of the Aq-A convergence series versus the (enclosed) relaxation time, $t_{\text {relax }}$, expressed in units of the circular orbit period at the virial radius, $t_{\text {circ }}\left(r_{200}\right)$. Deviations are measured relative to the highest resolution halo, Aq-A-1. Note that departures from convergence for all simulations are similar when expressed this way, indicating that $t_{\text {relax }}$ is the main parameter determining convergence. Solid circles mark the location of the convergence criterion proposed by $\mathrm{P} 03$. Note that $V_{c}$ estimates converge there to about $10 \%$. A stricter convergence criterion, e.g., $2.5 \%$ convergence in $V_{c}$, is achieved at larger radii, where $t_{\text {relax }} \sim 7 t_{\text {circ }}\left(r_{200}\right)$ (right vertical line). Bottom panel: Relaxation time versus radius for all five Aq-A simulations. Arrows indicate $h_{s}=2.8 \epsilon_{G}$, the lengthscale where pairwise interactions become Newtonian. grangian region surrounding the target halo, and a lowresolution region (the rest of the box), which is represented with a smaller number of particles with mass increasing with distance to the target halo. We have carefully designed the geometry of the high-resolution region in order to avoid contamination of the halo by massive low-resolution particles. Typically, about $30 \%$ of particles in the high-resolution region end up in the virialized region of the final halo, and no higher mass particles end up within the virial radius of the final halo.

Table 1 lists some basic information about each simulation. This includes a symbolic simulation name, the particle mass in the high-resolution region, $m_{p}$, the gravitational softening length, $\epsilon_{G}$, the virial radius ${ }^{\star}, r_{200}$, as well as the total mass, $M_{200}$ and the total number of particles, $N_{200}$, enclosed within $r_{200}$. Other structural parameters of interest include the location of the peak in the circular velocity profile, specified by $r_{\max }$ and $V_{\max }$, as well as that of the velocity dispersion profile $\left(\sigma_{\max }\right.$ and $\left.r\left(\sigma_{\max }\right)\right) . \sigma_{\text {host }}$ indicates the $1 \mathrm{D}$ rms velocity of the main halo within $r_{200}$ (excluding substructures).

Table 1 lists only information on the halos used in this paper. A more complete list of numerical parameters may be found in Springel et al. (2008b). One of our halos, labeled Aq-A, has been resimulated 5 times, spanning a factor of $\sim 2000$ in particle mass. Our naming convention uses the tags "Aq-A" through "Aq-F" to refer to each of the six Aquarius halos. An additional suffix "1" to "5" denotes the resolution level. "Aq-A-1" is our highest resolution calculation: it follows the surroundings of Aq-A with $\sim 4.4$ billion particles, $\sim 1.1$ billion of which end up within $r_{200}$. We have level-2 simulations of all 6 halos, corresponding to between 100 and 200 million particles per halo (within $r_{200}$ ). The softening parameters of each simulation adopt the "optimal" softening recommendation of $\mathrm{P} 03$, which aims to balance the number of timesteps required for accurate integration whilst minimizing the loss of spatial resolution.

\subsection{Radial Profiles}

Our analysis uses spherically-averaged profiles of the basic dynamical properties describing the structure of $\Lambda \mathrm{CDM}$ halos: the density, circular velocity, velocity dispersion, and anisotropy profiles. Typically, these are computed in 50 spherical shells equally spaced in $\log _{10} r$ (where $r$ is the distance to the halo center), and spanning the range $1.5 \times 10^{-4}<r / r_{200}<3$. (When different choices for either the number of bins or the radial range are made, this is stated explicitly in the analysis below.) These concentric

* We define the virial mass of a halo, $M_{200}$, as that contained within a sphere of mean density $200 \times \rho_{\text {crit }}$. The virial mass defines implicitly the virial radius, $r_{200}$, and virial velocity, $V_{200}=$ $\left(G M_{200} / r_{200}\right)^{1 / 2}$, of a halo, respectively. We note that other definitions of "virial radius" have been used in the literature; the most popular of the alternatives adopts a density contrast (relative to critical) of $\Delta \approx 178 \Omega_{\mathrm{m}}^{0.45} \sim 100$ (for our adopted cosmological parameters, see Eke et al. (1996)). We shall refer to these alternative choices, where appropriate, with a subscript indicating the value of $\Delta$; i.e., $r_{50}$ would be the virial radius obtained assuming $\Delta=50$, and so an enclosed density 200 times the mean cosmic value. 

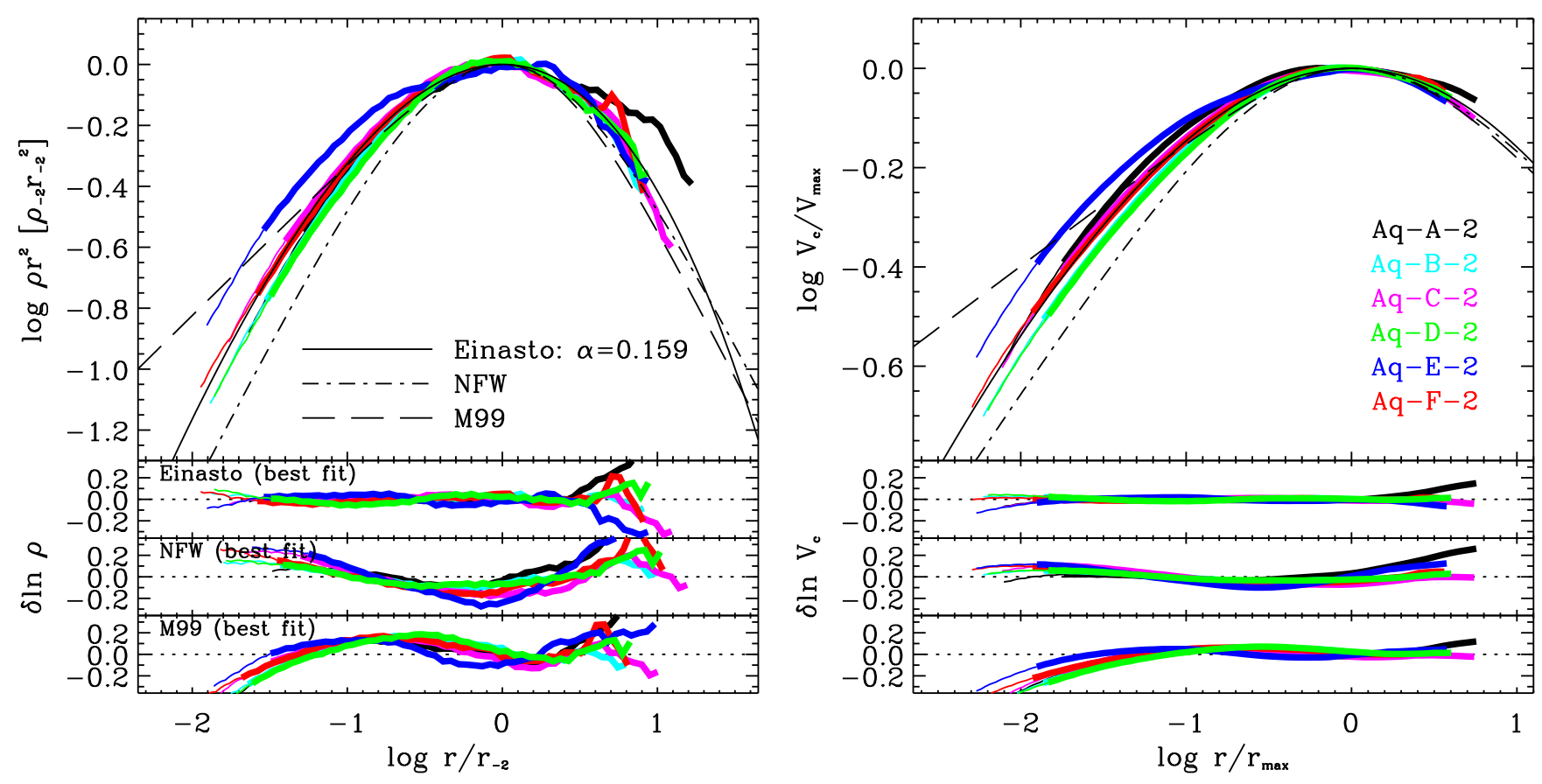

Figure 3. Left: Spherically-averaged density profiles of all level-2 Aquarius halos. Density estimates have been multiplied by $r^{2}$ in order to emphasize details in the comparison. Radii have been scaled to $r_{-2}$, the radius where the logarithmic slope has the "isothermal" value, -2 . Thick lines show the profiles from $r_{\text {conv }}^{(7)}$ outward; thin lines extend inward to $r_{\text {conv }}^{(1)}$. For comparison, we also show the NFW and M99 profiles, which are fixed in these scaled units. This scaling makes clear that the inner profiles curve inward more gradually than NFW, and are substantially shallower than predicted by M99. The bottom panels show residuals from the best fits (i.e., with the radial scaling free) to the profiles using various fitting formulae (Sec. 3.2). Note that the Einasto formula fits all profiles well, especially in the inner regions. The shape parameter, $\alpha$, varies significantly from halo to halo, indicating that the profiles are not strictly self-similar: no simple physical rescaling can match one halo onto another. The NFW formula is also able to reproduce the inner profiles quite well, although the slight mismatch in profile shapes leads to deviations that increase inward and are maximal at the innermost resolved point. The steeply-cusped Moore profile gives the poorest fits. Right: Same as left, but for the circular velocity profiles, scaled to match the peak of each profile. This cumulative measure removes the bumps and wiggles induced by substructures and confirms the lack of self-similarity apparent in the left panel.

shells are centered at the location of the particle identified by the SUBFIND algorithm (Springel et al., 2001) as having the minimum gravitational potential. Extensive tests show that this procedure identifies the region where the local density of the main subsystem of each halo peaks, and is coincident in most cases (except perhaps major ongoing mergers between comparable-mass halos) with the results of other methods, such as the "shrinking sphere" method discussed by P03.

The mass density in each radial bin is estimated as the dark mass in the bin divided by its volume, and assigned to a radius corresponding to the bin center. Circular velocities are computed by adding up the mass of each bin plus all interior ones, and assigned to the radius corresponding to the outer edge of the bin. The construction of velocity dispersion and anisotropy profiles is described in detail in Sec. 4.1. When differentiation is necessary, such as when computing the logarithmic slopes shown in Figs. 5 and 6, we use a simple 3 -point Lagrangian interpolation to perform the numerical differentiation (as implemented by the DERIV subroutine of the IDL software package).

\section{MASS PROFILES}

\subsection{Numerical Convergence}

We begin our study of the mass profile by using our series of re-simulations of the Aq-A halo in order to assess the radial range where numerical convergence is achieved. Figure 1 shows the mass profile of the five Aq-A resimulations; the left panels show the spherically-averaged density profile (multiplied by $r^{2}$ in order to emphasize small departures); the right panels the corresponding circular velocity profile. Lines of different colours correspond to different resimulations, as labeled. Arrows indicate $h_{s}=2.8 \epsilon_{G}$, the lengthscale where softened pairwise interactions become fully Newtonian.

This figure demonstrates the striking numerical convergence achieved in our re-simulations. Outside some characteristic radius (which we discuss below), all the profiles are essentially indistinguishable from each other, even down to details such as "bumps" in the outer regions caused by the presence of substructure. As discussed by Springel et al. (2008b), this reflects the high quality of the numerical integration of GADGET-3 and the careful approach we have taken to building our initial conditions; indeed, the Aq-A resimulations not only reproduce faithfully the properties 
of the main halo, but even the mass, location and internal structure of most major substructures.

Inevitably, near the centre the mass profiles diverge as a consequence of numerical limitations. Each profile is plotted down to the "convergence radius" proposed by P03. These authors demonstrate that deviations from convergence depend (for appropriate choices of other numerical parameters) solely on the number of particles, and scale roughly with the collisional "relaxation" time, $t_{\text {relax }}$. Expressed in units of the circular orbit timescale at $r_{200}$ (which is of the order of the age of the Universe), $\kappa=t_{\text {relax }} / t_{\text {circ }}\left(r_{200}\right)$, the relaxation time may be written as:

$\kappa(r)=\frac{N}{8 \ln N} \frac{r / V_{c}}{r_{200} / V_{200}}=\frac{\sqrt{200}}{8} \frac{N(r)}{\ln N(r)}\left(\frac{\bar{\rho}(r)}{\rho_{\text {crit }}}\right)^{-1 / 2}$,

where $N=N(r)$ is the enclosed number of particles and $\bar{\rho}(r)$ is the mean enclosed density within $r$.

According to P03, deviations of roughly $10 \%$ are expected in the $V_{c}$ profile where $\kappa \approx 1$, and they adopted this condition to define the convergence radius, $r_{\text {conv }}$. Stricter convergence demands larger values of $\kappa$, and we shall use a superscript on $r_{\text {conv }}$ to denote the value of $\kappa$ adopted for its definition. For instance, $r_{\mathrm{conv}}^{(1)}=r_{\mathrm{conv}}^{(\kappa=1)}$ corresponds to $\kappa=1$.

Profiles in Fig. 1 are thus plotted in the range $\left[r_{\text {conv }}^{(1)}\right.$, $\left.3 r_{200}\right]$. As shown in the bottom right panel, this inner radius indeed corresponds to the point where systematic deviations in $V_{c}(r)$ reach $\sim 10 \%$. It is also clear from this figure that convergence in the local density profile is always much easier to achieve, so concentrating our analysis on the enclosed mass profile, or on the circular velocity, is a conservative approach.

Although each halo converges over a different radial range, the departures from convergence are all similar when expressed in terms of $\kappa$. This is shown in the top panel in Fig. 2, where differences in $V_{c}$ from our highest-resolution halo, Aq-A-1, are shown as a function of $\kappa$ for the other AqA resimulations. Deviations of $\sim 10 \%$ are typical at $\kappa=1$; convergence to better than $\sim 2.5 \%$, on the other hand, requires $\kappa \approx 7$ (indicated by the right dashed vertical line).

We may use these results to estimate convergence radii for our highest resolution run, Aq-A-1: its $V_{c}$ profile converges to better than $10 \%$ for radii $r>r_{\mathrm{conv}}^{(1)}=112 h^{-1}$ pc; $2.5 \%$ convergence or better is expected for $r>r_{\text {conv }}^{(7)}=$ $253 h^{-1}$ pc (see bottom panel of Fig. 2). Convergence radii for various values of $\kappa$ are listed in Table 2 for each simulated halo.

\subsection{Fitting formulae}

The fitting formulae we have used to describe the mass profile of our simulated halos are the following: (i) The NFW profile, given by

$\rho(r)=\frac{\rho_{s}}{\left(r / r_{s}\right)\left(1+r / r_{s}\right)^{2}}$,

(ii) the modification to the NFW profile proposed by M99,

$\rho(r)=\frac{\rho_{M}}{\left(r / r_{M}\right)^{1.5}\left[1+\left(r / r_{M}\right)^{1.5}\right]}$,

and (iii) the Einasto profile,

$\ln \left(\rho(r) / \rho_{-2}\right)=(-2 / \alpha)\left[\left(r / r_{-2}\right)^{\alpha}-1\right]$

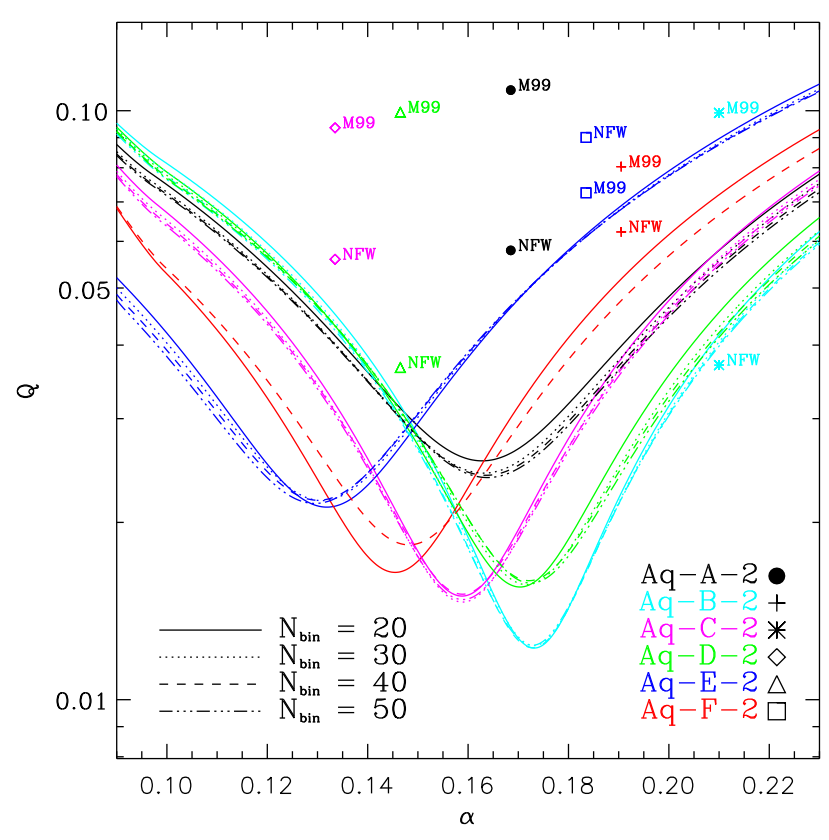

Figure 4. Minimum- $Q$ values as a function of the Einasto parameter $\alpha$ for best fits to all level-2 halo profiles in the radial range $0.01<r / r_{-2}<5$. Colors identify different halos, and line types the number of bins chosen for the profile. The minimum- $Q$ values obtained for NFW and M99 best fits are also shown, and are plotted at arbitrary values of $\alpha$ for clarity. Note that Einasto fits are consistently better than NFW which are consistently better than M99, and that a significant improvement in $Q$ is obtained when letting $\alpha$ vary in the Einasto formula. $Q$ is approximately independent of the number of bins used in the profile, and is minimized for different values of $\alpha$ for each individual halo. See text for further details.

Because each of these formulae defines the characteristic parameters in a slightly different way, we choose to reparametrise them in terms of $r_{-2}$ and $\rho_{-2} \equiv \rho\left(r_{-2}\right)$, which identify the "peak" of the $r^{2} \rho$ profile shown in the left panel of Fig. 1. This marks the radius where the logarithmic slope of the profile, $\gamma(r)=-d \ln \rho / d \ln r$, equals the isothermal value, $\gamma=2$.

The characteristic radius, $r_{-2}$, is a well-defined scalelength which is relatively easy to identify in each halo without resorting to any particular fitting formula. In practice, we determine $r_{-2}$ by computing the logarithmic slope profile, $\gamma(r)$, and identifying where a low-order polynomial fit to it intersects the isothermal value. Each $r^{2} \rho$ profile is then visually inspected in order to ensure that $r_{-2}$ corresponds to the main peak of the profile, and that it is not unduly influenced by secondary peaks that arise as the result of substructure. (See the left panel of Fig. 1.) Table 2 lists $r_{-2}$ and $\rho_{-2}$ for all our simulated halos. Note that for the NFW profile, $r_{-2}=r_{s}$ and $\rho_{-2}=\rho_{s} / 4$, while for the Moore profile, $\rho_{-2}=(4 / 3) \rho_{M}$ and $r_{-2}=2^{-2 / 3} r_{M}$.

We note that, unlike NFW or M99, when $\alpha$ is allowed to vary freely the Einasto profile is a 3-parameter fitting formula. This is not, of course, the only possible extension of NFW-like profiles which allows for a variable shape with the aid of an extra free parameter. For example, 


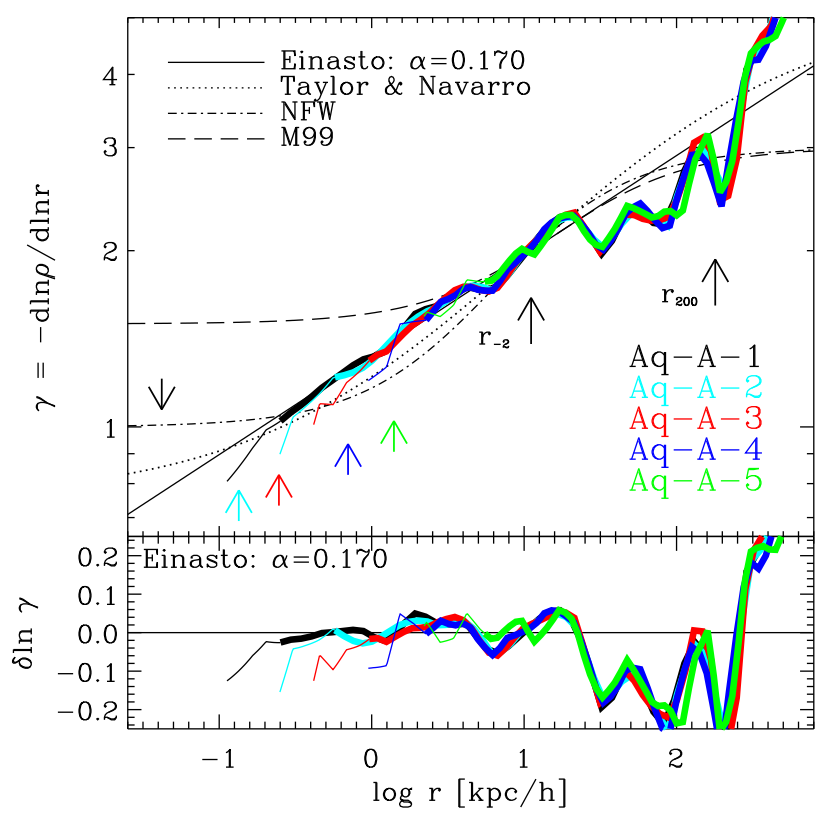

Figure 5. Logarithmic slope of the density profile as a function of radius for our Aq-A convergence series. As in other plots, thick lines show results for $r>r_{\mathrm{conv}}^{(7)}$, thin lines extend the profiles down to the less strict convergence radius $r_{\text {conv }}^{(1)}$. Comparison shows that excellent numerical convergence for the slope is achieved down to a radius intermediate between these two convergence radii. Applied to the highest-resolution Aq-A-1 simulation, this implies that the slope is shallower than the asymptotic value of the NFW profile $\left(r^{-1}\right)$ in the inner regions. We see no sign of convergence to an asymptotic inner power-law. Instead, the profiles get shallower toward the centre as predicted by the Einasto formula (a straight line in this plot). The "critical solution" of Taylor \& Navarro (2001) (which has a $r^{-0.75}$ asymptotic inner cusp) does better than NFW but not as well as Einasto in reproducing the inner profile of the halo.

Merritt et al. (2006) compared N-body halos with the 3parameter Einasto formula, as well as with the anisotropic model of Dehnen \& McLaughlin (2005) and with the deprojected Sersic (1968) model of Prugniel \& Simien (1997). Merritt et al. conclude that, overall, Einasto's formula performs best. Therefore, we adopt it here for the rest of our analysis, although we do not exclude the possibility that other 3-parameter formulae may perform at least as well as Einasto's. A full exploration of this issue is beyond the scope of this paper.

\subsection{Fitting procedure}

Best-fit parameters are found by minimizing the deviation between model and simulation across all bins in a specified radial range. In the case of the density profile, the best fit is found by minimizing the figure-of-merit function, $Q^{2}$, defined by

$Q^{2}=\frac{1}{N_{\text {bins }}} \sum_{i=1}^{N_{\text {bins }}}\left(\ln \rho_{i}-\ln \rho_{i}^{\text {model }}\right)^{2}$

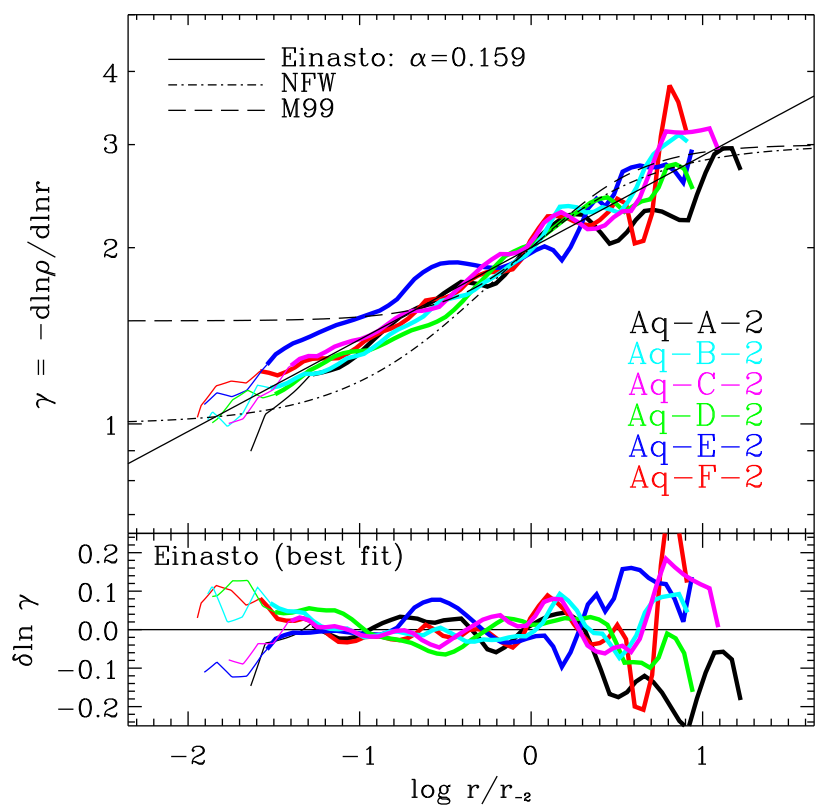

Figure 6. As Fig. 5, but for all level-2 resolution Aquarius halos, after scaling radii to $r_{-2}$.

This function provides an intuitively simple measure of the level of disagreement between simulated and model profiles. It is dimensionless; it weights different radii logarithmically; and, for given radial range, $Q^{2}$ is approximately independent of the number of bins used in the profile. Thus, minimizing $Q^{2}$ yields for each halo well-defined estimates of a model's best-fit parameters. Note that when $Q$ is small, it is just the rms fractional deviation of the data from the model.

It is less clear how to define a goodness-of-fit measure associated with $Q^{2}$ and, consequently, how to assign statistically-meaningful confidence intervals to the best-fit parameter values. This difficulty arises because, at the very high resolution of the simulations analyzed here, discreteness noise in the binned density estimates is negligible. The figure of merit of a fit therefore depends not only on how faithfully a model approximates a halo but also on the presence of individual halo features that no simple fitting formula can hope to reproduce. These distinct features are present on small scales (substructure) and large scales (such as streams, asphericity, and other relics of each halo's specific assembly history; see, e.g., Vogelsberger et al., 2009). As a result, binto-bin residuals are distinctly non-Gaussian and highly correlated, precluding the use of simple statistical tools such as the $\chi^{2}$ distribution in order to assess goodness of fit.

Assessing the acceptability of various $Q$ values would require the definition of a detailed statistical model in order to measure reliably the departures of individual halos from a smooth profile whose average shape (and scatter) could be obtained directly by averaging various numerical realizations of halos of the same mass. Unfortunately, such procedure is unlikely to be robust with only 6 halos in our sample.

Therefore, we limit our analysis to comparing the minimum- $Q$ values obtained with various formulae, and to 
Navarro et al. .

discussing how $Q$ changes as the fitting parameters are varied. The actual value of $Q$ is, after all, a reliable and objective measure of the average per-bin deviation from a particular model. As we discuss below, this is, in many cases, enough to prefer unequivocally one fitting formula over another and to make a compelling case for the need of an extra parameter in the fit.

\subsection{Einasto vs NFW vs M99}

The left panel of Fig. 3 compares the density profiles of all six level-2 Aquarius halos, after scaling radii to $r_{-2}$ and densities to $\rho_{-2}$. The right-hand panel shows the circular velocity profiles, scaled in an analogous manner to match the peak of the profile, identified by $r_{\max }$ and $V_{\max }$. In these scaled units, the fitting formulae introduced in Sec. 3.2 are curves of fixed shape and normalization, as shown by the thin solid, dashed, and dot-dashed curves in Fig. 3. (The Einasto curve adopts $\alpha=0.159$ in this figure.)

Comparison with the simulations (thick curves) indicates that there is a clear mismatch between the shape of the halo profiles and those of the NFW and M99 fitting formulae. This is not just a result of enforcing the $r_{-2}-\rho_{-2}$ scaling. We illustrate this by showing, in the two bottom panels of Fig. 3, residuals from best fits obtained by adjusting both fit parameters of the NFW and M99 profiles $\left(r_{-2}\right.$ and $\rho_{-2}$ ) in order to minimize $Q^{2}$. (The radial range chosen for these fits is $r_{\text {conv }}^{(1)}<r<0.5 r_{200}$.) Note the "S" shape in the residuals, which are largest (and increasing) at the innermost radius of the profile. Because of the shape mismatch, extrapolating either the NFW or M99 fits further inwards, to regions less well resolved numerically, is almost guaranteed to incur substantial error.

The large-scale radial trend of the residuals from the best Einasto fits (middle panels of Fig. 3), on the other hand, is rather weak, suggesting that the shape of the simulated halo profiles are much better accommodated by this formula. This is not just a result of the extra shape parameter in the Einasto formula: even when keeping $\alpha$ fixed to a single value, residuals are smaller and have less radial structure than those from either NFW or M99.

We show this in Fig. 4, where we plot the minimum$Q\left(Q_{\min }\right)$ values of the best Einasto fits for all six level-2 Aquarius halos, as a function of the shape parameter $\alpha$. For given value of $\alpha$ the remaining two free parameters of the Einasto formula are allowed to vary in order to minimize $Q^{2}$. Different line types correspond to different numbers of bins used to construct the profile (from 20 to 50), chosen to span in all cases the same radial range, $0.01<r / r_{-2}<5$, a factor of 500 in radius. Minimum- $Q$ values are computed using a similar procedure for the NFW and M99 formulae, and are shown, for each halo, with symbols of corresponding colour.

In terms of $Q_{\min }$, Einasto fits are consistently superior to NFW or M99, whether or not the $\alpha$ parameter is adjusted freely. For example, for fixed $\alpha=0.15$, all Einasto best fits have minimum- $Q$ values below $\sim 0.03$. For comparison, best NFW and M99 fits have an average $\left\langle Q_{\min }\right\rangle \sim 0.06$ and 0.095 , respectively. These numbers correspond to $N_{\text {bins }}=20$, but they are rather insensitive to $N_{\text {bins }}$, as may be judged from the small difference between the various lines corresponding to each halo in Fig. 4.

We emphasize that, although the improvement obtained with Einasto's formula is significant, NFW fits are still excellent, with a typical rms deviation of just $\sim 6 \%$ over a range of 500 in radius. The use of the NFW formula may thus be justified for applications where this level of accuracy is sufficient over this radial range.

When $\alpha$ is adjusted as a free parameter, $\left\langle Q_{\min }\right\rangle \sim 0.018$ for Einasto fits. Furthermore, there is, for each halo, a well defined value of $\alpha$ that yields an absolute minimum in $Q$. The $Q$-dependence on $\alpha$ about this minimum is roughly symmetric and, as expected, nearly independent of the number of bins used in the profile. The minimum in $Q$ is sharp; a shift of just 0.015 in $\alpha$ typically leads to an increase of $\sim 50 \%$ in $Q$ around the minimum. Given that the value of $\alpha$ that minimizes $Q$ varies from 0.130 for Aq-E-2 to 0.173 for Aq-B-2, we conclude that the improvement obtained when allowing $\alpha$ to vary is significant. We quote nominal "error bars" for $\alpha$ in Table 2 that bracket the interval where $Q$ deviates by less than $50 \%$ from the absolute minimum in Fig. 4.

\subsection{Self-similarity?}

The need for a variable $\alpha$ discussed above illustrates one of our main findings: namely, that the mass profiles of our Aquarius halos are not strictly self similar. The shapes of the profiles are subtly but significantly different from each other, and no rescaling can match one exactly to another. Halo Aq-E-2 provides the most striking example, deviating from halo Aq-D-2, for example, by almost a factor of 2 in density at $\sim 0.03 r_{-2}$. The same differences in mass profile shape are also easily appreciated in the scaled circular velocity profiles, which indicate that the departures from similarity are genuine and not just caused by inaccuracies in the scaling or by the "bumps and wiggles" caused by unrelaxed tidal debris and substructure.

We have verified this further by performing the same analysis after removing bound substructure clumps identified by SUBFIND: the same conclusion applies to the "cleaned" profiles of the main smooth halo. With hindsight, this is perhaps not too surprising. Bound substructures do not amount to more than $\sim 10 \%$ of the halo mass (Springel et al., 2008b), and therefore cannot alter the results discussed above.

We have also checked that the differences in $\alpha$ are not caused by transient departures from equilibrium or numerical resolution: the same qualitative trends, and indeed very similar $\alpha$ values, are seen at earlier times and in runs with fewer particles. There also seems to be little correlation between $\alpha$ and the overall triaxiality of the system; however, we shall only deal here with spherically-averaged profiles, and defer a detailed study of departures from sphericity to a later paper.

Although the departures from similarity appear significant, we must also emphasize that they are rather subtle, and are only clearly evident because of the large radial range resolved by our simulations, about three decades in radius within the virialized region of a halo. Simulations with more limited numerical resolution have hinted at this but had difficulty making such a compelling case for non-similarity (see, 


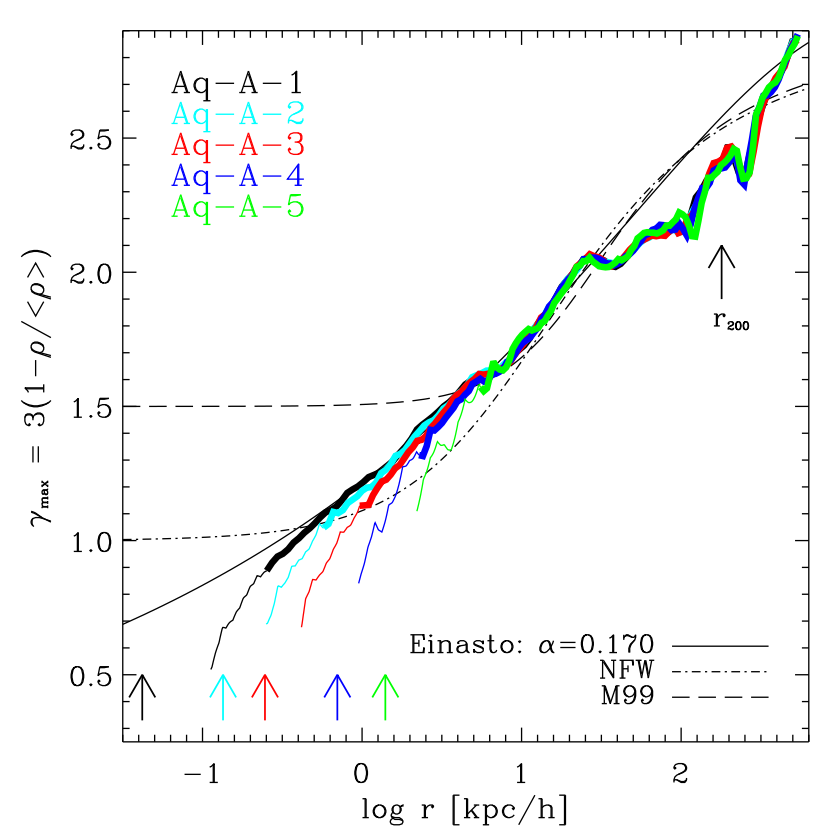

Figure 7. Maximum value of the asymptotic inner slope of the density cusp, as a function of radius for our Aq-A convergence series. Excellent numerical convergence is achieved at radii comparable to $r_{\text {conv }}^{(7)}$ (the inner limit of the thick lines; thin lines extend down to $\left.r_{\text {conv }}^{(1)}\right)$. This shows that there is not enough mass near the centre of Aq-A to sustain a cusp steeper than $\rho \propto r^{-0.9 \pm 0.1}$. Arrows are as in Fig. 1.

e.g., Navarro et al., 2004; Merritt et al., 2005; Stoehr, 2006; Merritt et al., 2006).

\subsection{The Cusp}

It is clear from the residuals in the bottom panels of Fig. 3 that, near the centre, the M99 profile approximates the simulated halos more poorly than either NFW or Einasto. The weak performance of the M99 formula may be traced to its steep asymptotic inner slope, $\rho \propto r^{-1.5}$. Indeed, all six Aquarius halos have measured slopes in the inner regions that are substantially shallower than -1.5 . This is shown in Figs. 5 and 6, where the thick portion of each curve corresponds to $r>r_{\text {conv }}^{(7)}$ and the innermost point plotted to $r_{\text {conv }}^{(1)}$. In all cases, the logarithmic slopes converge well inside $r_{\mathrm{conv}}^{(7)}$, and only minor deviations may be seen at radii beyond $r_{\mathrm{conv}}^{(1)}$.

Interestingly, the slope of the Aq-A-1 profile at $r=r_{\mathrm{conv}}^{(7)}$ is exactly -1 , and becomes shallower inward, so it is clear that at least for this halo we are able to resolve a region where the dark matter profile has become shallower than -1 , the asymptotic value of the NFW profile. Fig. 6 shows the radial dependence of the logarithmic slope for all six level-2 halos and confirms the general applicability of the Aq-A results: the measured slopes of all halos approach -1 (and are certainly shallower than -1.5 ) at the innermost resolved point.

Figs. 5 and 6 also make clear that there is no sign that the profiles are approaching power-law behaviour near

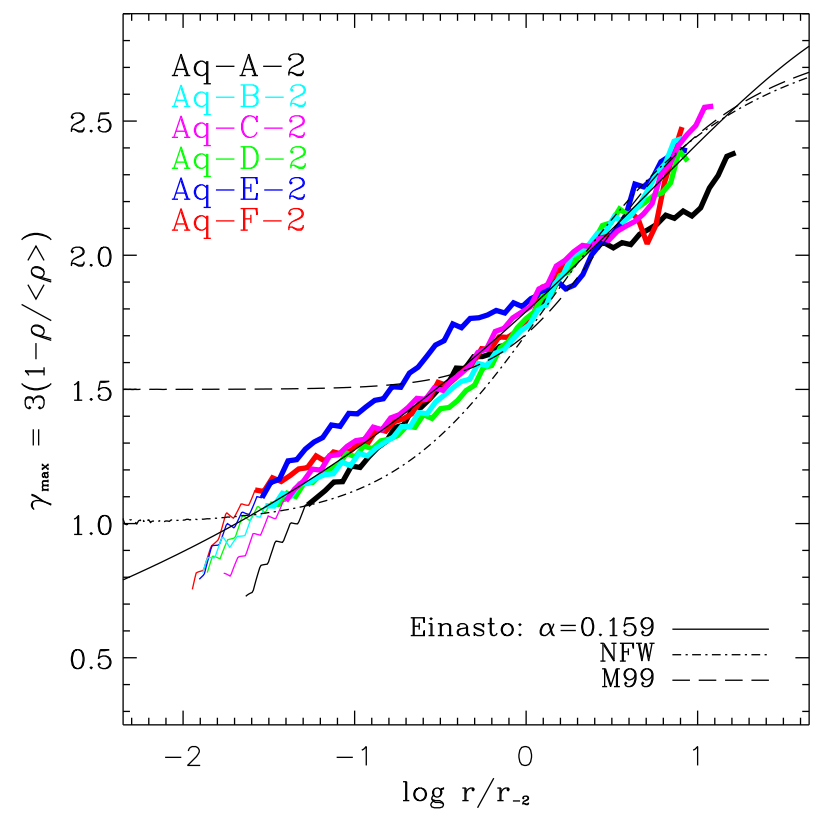

Figure 8. As Fig. 7, but for our six level-2 Aquarius halos. Results are similar in all cases and rule out cusps steeper than $r^{-1}$ for $\Lambda$ CDM halos.

the centre: they keep getting shallower to the innermost resolved radius. This behaviour is well captured by the Einasto model, where the logarithmic slope is simply a power-law of radius, $d \ln \rho / d \ln r \propto r^{\alpha}$. Our results thus rule out recent claims of cusps as steep as $r^{-1.2}$ in typical $\Lambda$ CDM halos (Diemand et al., 2004, 2005, 2008).

This conclusion is unlikely to depend on the details of our profile construction and/or fitting procedures. Indeed, as we show in the next subsection, there is actually not enough mass within the innermost resolved radius to allow for a cusp as steep as $r^{-1.2}$. Recent work by Stadel et al. (2008), also based on very high-resolution simulations, agrees with our present conclusions, and argues for asymptotic inner slopes shallower than -1 , as previously suggested by Navarro et al. (2004).

\subsection{The Asymptotic Inner Slope}

The results presented above do not preclude the possibility that a shallow power-law cusp may be present in the innermost regions which are still unresolved in our simulations. It is therefore interesting to estimate the maximum value that the slope of such a cusp may take. This is constrained, at any radius, by the total enclosed mass and the local value of the spherically averaged density: slopes steeper than $\gamma_{\max }$ require more mass than is available within that radius. This constraint assumes only that the logarithmic slope is monotonic with radius and that the halo is not hollow. It is then straightforward to show that the maximum possible inner asymptotic slope is $\gamma_{\max }=3(1-\rho(r) / \bar{\rho}(r))$, where $\bar{\rho}(r)$ is the mean density enclosed within $r$. Evaluated at the innermost radius where both local density and enclosed mass (or, equivalently, circular velocity) have converged, this quan- 
tity provides an important constraint on the density profile at radii that remain unresolved even in our best simulations.

We show this parameter as a function of radius for our Aq-A convergence series in Fig. 7. This figure shows that $\gamma_{\max }$ converges to better than 0.1 for $r>r_{\text {conv }}^{(7)}$ (the innermost point of the thick portion of the profiles). Our data for Aq-A thus indicates that there is not enough mass in the unresolved region to support a cusp steeper than $r^{-0.9 \pm 0.1}$. Fig. 8 shows that the results for Aq-A are not exceptional: all our level-2 Aquarius halos suggest maximum possible asymptotic slopes of about -1 .

\section{DYNAMICAL PROFILES}

\subsection{Velocity Dispersion Structure}

Fig. 9 shows velocity dispersion and anisotropy profiles for our Aq-A series and demonstrates that the excellent numerical convergence of our simulations extends to their velocity dispersion structure. The velocity dispersion (squared) is computed simply as twice the specific kinetic energy in each spherical shell and the anisotropy as $\beta=1-\sigma_{t}^{2} /\left(2 \sigma_{r}^{2}\right)$, where $\sigma_{t}^{2}$ and $\sigma_{r}^{2}$ are the (squared) velocity dispersion in tangential and radial motions, respectively. Besides numerical convergence, the panels in this figure illustrate two important points. The first concerns the shape of the velocity dispersion profiles (left panel in Fig. 9), which is remarkably similar to that of the $r^{2} \rho$ profiles shown in Fig. 1. This coincidence suggests an intimate connection between density and velocity dispersion, which we explore in more detail in Sec. 4.4. The second point concerns the anisotropy profile, which is clearly non-monotonic. It is nearly isotropic at the centre, becomes radially anisotropic at intermediate radii, but the dominance of radial motions decreases again near the virial radius. As shown in Fig. 10, these properties appear to be rather general, since all six Aquarius halos have non-monotonic anisotropy profiles and similar velocity dispersion profile shapes.

\subsection{Self-similarity?}

Fig. 10 also demonstrates a clear lack of self-similarity in the structure of the simulated halos. We have chosen to emphasize this by rescaling all profiles so as to match the peak of the $\sigma(r)$ curve, which occurs at $r\left(\sigma_{\max }\right)$. This scaling demonstrates that, as with the density profiles, the shape of the $\sigma(r)$ profiles differs subtly but significantly amongst halos. We have checked that these differences in shape are not due to bound subhalos; removing all the subhalos identified by our SUBFIND algorithm and recalculating the dispersion and anisotropy profiles results in only rather minor changes

The most striking case is again that of halo Aq-E-2 (blue curve), whose $\sigma(r)$ profile is much broader than the others. Recall that this halo also stands out in Fig. 3 as having an unusually broad $r^{2} \rho$ profile. Halo Aq-E-2 also has an unusual velocity anisotropy profile, with less predominance of radial motions than the rest of the series. The departures from similarity in mass and velocity structure therefore seem closely linked, suggesting that these halos may share a common property that combines density and velocity dispersion. We explore this in Sec. 4.4 below.

\subsection{Anisotropy-slope relation}

We may use the results of the previous subsection to assess recent claims by Hansen \& Moore (2006) of a general connection between the local values of logarithmic slope, $\gamma$, and the velocity anisotropy, $\beta$. We show this in Fig. 11, where we plot $\beta$ vs $\gamma$ for all level-2 Aquarius halos. Open circles correspond to the inner regions of the halo $\left(r_{\text {conv }}^{(1)}<r<r_{-2}\right)$ whereas filled circles correspond to the outer regions $\left(r_{-2}<\right.$ $\left.r<r_{200}\right)$. As in other figures, different colours correspond to the different Aquarius halos. The relation proposed by Hansen \& Moore is shown by a dashed line and accounts reasonably well (albeit not perfectly) for our data in the inner regions where both the anisotropy and the logarithmic slope are monotonic functions of $r$.

However, there are large departures from this relation in the outer regions, where the density profile steepens further but the velocity ellipsoid tends to become less anisotropic. The failure of the Hansen \& Moore relation in the outer regions is not unexpected since $\gamma$, unlike $\beta$, is monotonic with radius. We conclude that, if a simple relation links anisotropy and slope, it can only hold in the inner regions of halos.

\subsection{The Phase-Space Density Profile}

The similarity in shape between the $\sigma^{2}$ and $r^{2} \rho$ profiles highlighted above suggests that there may be a simple scaling between densities and velocity dispersions in halos. This is best appreciated by considering the quantity $\rho / \sigma^{3}$, which, for dimensional reasons, we shall call the pseudo-phase-space density, although it is important to realise that it is not the true coarse-grained phase-space density at the resolution of our simulations, or even the average of this quantity in spherical shells. For consistency with the rest of our analysis, we calculate $\rho / \sigma^{3}$ directly from the estimates of $\rho$ and $\sigma$ computed in concentric spherical shells.

Fig. 12 shows the $\rho / \sigma^{3}$ profile for our Aq-A convergence series. As noted by Taylor \& Navarro (2001), the profile of this quantity is remarkably well approximated by a power-law. More remarkable still is the fact that the power law is indistinguishable from that predicted by the similarity solution of Bertschinger (1985) for infall onto a point mass in an otherwise unperturbed Einstein-de Sitter universe, $\rho / \sigma^{3} \propto r^{-1.875}$ (dot-dashed line in Fig. 12). This solution is spherically symmetric, involves purely radial motions, and is violently dynamically unstable, so its relevance to $\Lambda$ CDM halos is far from clear. The residuals in the bottom panel of Fig. 12 are deviations from a Bertschinger law matched within the characteristic radius $r_{-2}$, where substructure bumps and wiggles are minimal.

Note that, although there is only one free parameter in this fit (the vertical scaling), the residuals do not exceed $\sim 20 \%$ anywhere within the virial radius, even though substructures add significant noise to the dynamical measurements in the outskirts of the halo. Interestingly, the residuals increase when $\sigma_{r}$, the velocity dispersion in radial motions, is used in place of the full 3D rms velocity, $\sigma$,to estimate the "phase-space density". Thus, the $r^{-1.875}$ behaviour seems to concern the full kinetic energy content of each shell rather than just radial or tangential motions.

Fig. 13 shows that similar conclusions apply to the rest 

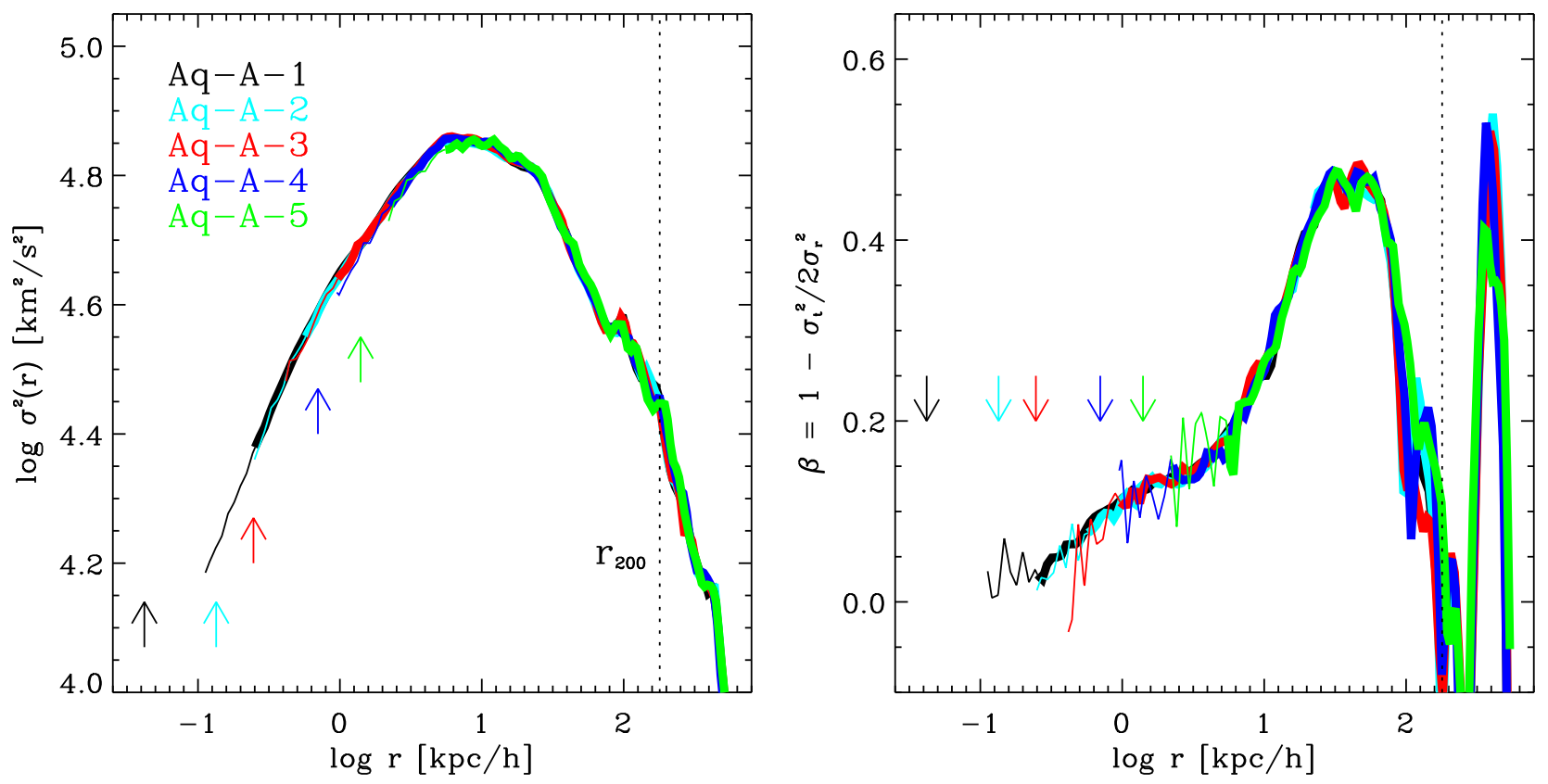

Figure 9. Left panel: Velocity dispersion profiles for our Aq-A convergence series. Arrows, line-types and colours are as in Fig. 1. Note the excellent numerical convergence. The shape of the velocity dispersion profile is remarkably similar to that of the $r^{2} \rho$ profile shown in Fig. 1, highlighting the intimate connection between the density and velocity dispersion profiles which is responsible for the power-law behaviour of the pseudo-phase-space density profile discussed in Sec. 4.4. Right panel: Anisotropy profiles for the Aq-A convergence series. Note the non-monotonic variation with radius: the halo is nearly isotropic near the centre, is dominated by radial motions at intermediate radii, but becomes markedly less anisotropic near the virial radius.
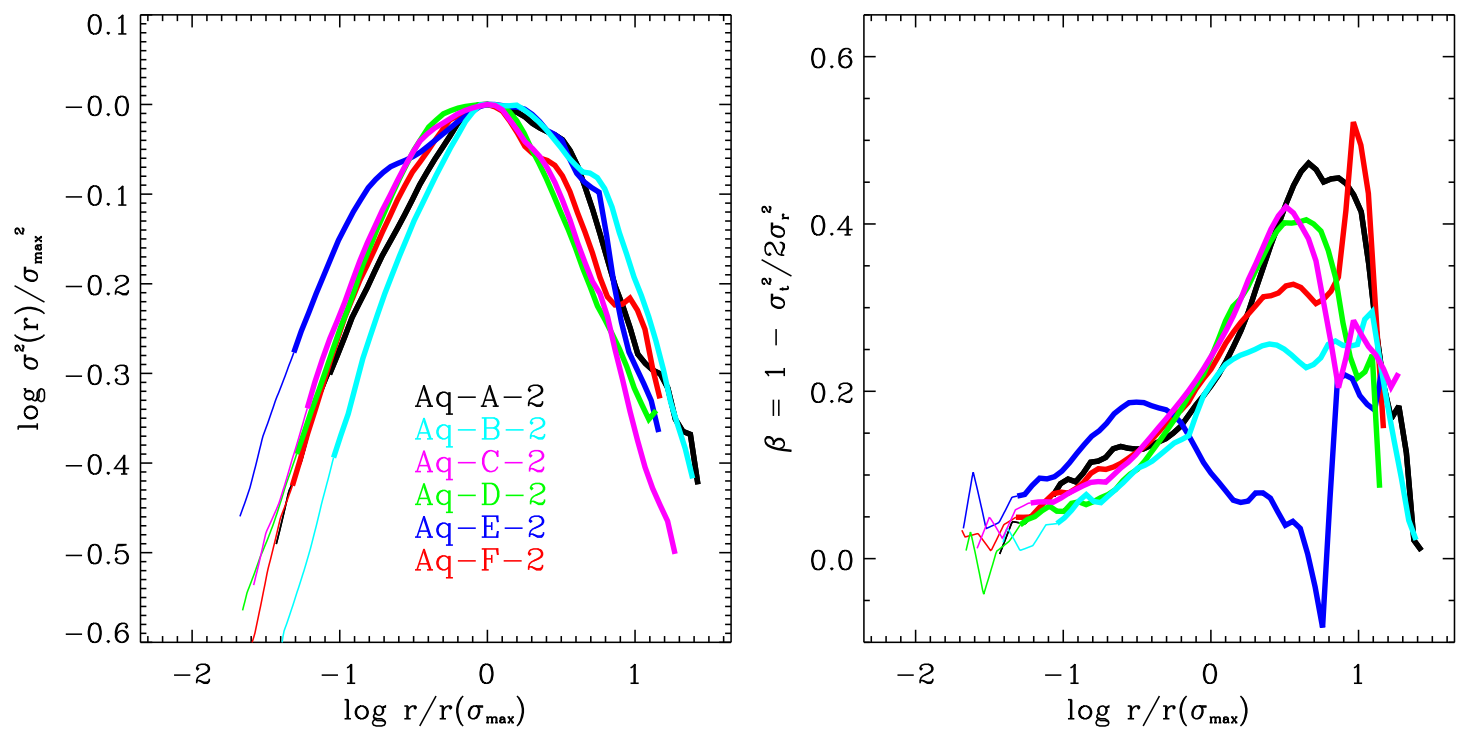

Figure 10. As Fig. 9, but for all six level-2 resolution Aquarius halos, scaled to match at the peak of the profile, identified by $\sigma_{\text {max }}$ and $r\left(\sigma_{\max }\right)$. This scaling highlights small but significant departures from similarity in the velocity dispersion structure of $\Lambda$ CDM halos. Note the correspondence in shape between the velocity dispersion and $r^{2} \rho$ profiles shown in Fig. 1, which reflects the "universal" pseudophase-space density profile of the halos (Fig. 13). Note also that the non-monotonic behaviour of the anisotropy highlighted in Fig. 9 is common to all six halos. 
of the Aquarius halos. Residuals from the Bertschinger law are small for all halos, and are typically larger when the radial velocity dispersion is used. Note that there is some "curvature" in the residual profiles, suggesting that a powerlaw is a good, but perhaps not perfect, description of the radial dependence of $\rho / \sigma^{3}$. We are currently investigating the origin of this curvature and plan to report on it in a future paper (Ludlow et al., in preparation).

A power-law radial dependence is approximately preserved when $\sigma_{r}$ is used, but the best fitting value of the exponent differs systematically from -1.875 . This may be seen in the bottom panels of Fig. 13, which show the residuals from the best fitting $\rho / \sigma^{3} \propto r^{\chi}$ law. The values of the best-fit exponent for both $\rho / \sigma^{3}$ and $\rho / \sigma_{r}^{3}$ ( $\chi$ and $\chi_{r}$, respectively) are listed in Table 2 .

Perhaps the most important result from Fig. 13 is that there seems to be very little scatter between halos when considering their $\rho / \sigma^{3}$ profiles. Take, for example, the case of halo Aq-E-2, which was a clear outlier in the density, velocity dispersion, and anisotropy profiles. When considering $\rho / \sigma^{3}$ this halo is unremarkable, and follows the Bertschinger law as closely as the others.

This shows that there is a sense in which $\Lambda$ CDM halos are nearly universal, but that universality does not extend to their density or velocity dispersion profiles separately, but rather only to their pseudo-phase-space density profile. This may appear a bold statement, and it certainly needs to be corroborated by future work, but it offers an intriguing perspective into the origin of the near-universal density profiles of halos, the meaning of the Einasto shape parameter, $\alpha$, and the provenance of their velocity dispersion structure. These issues deserve further investigation.

We end by noting that, although it is still not clear what leads to the power-law stratification of $\rho / \sigma^{3}$, these results may be used to place constraints on the structure of the central cusp, under the plausible (but admittedly unproven) assumption that the power-law behaviour of the phase-space density continues all the way to the centre. For example, Taylor \& Navarro (2001) used this assumption to show that, for isotropic systems, a power-law pseudo-phasespace density implies an inner density cusp with $\rho \propto r^{-0.75}$. This is certainly consistent with the results shown in Fig. 7, which only exclude cusps steeper than $r^{-0.9 \pm 0.1}$. However, as we show in Fig 5 , the detailed profile which they derive for an isotropic halo with Bertschinger's power-law $\rho / \sigma^{3}$ profile is a significantly worse fit to our numerical data than the Einasto profile.

The power-law behaviour of the pseudo phase-space density has been confirmed by a number of authors, and seems to be present even at early redshift (Vass et al., 2008). Interestingly, the average power-law exponent to the $\rho / \sigma_{r}^{3}$ profile is $\left\langle\chi_{r}\right\rangle \approx 1.97$, close to the "critical" 1.94 required by Dehnen \& McLaughlin (2005) to have a dynamical model that is well behaved at all radii. Simulations of even larger dynamic range seem required in order to explore the true asymptotic inner behaviour of the dynamical profile of a halo, if indeed there is any such asymptote.

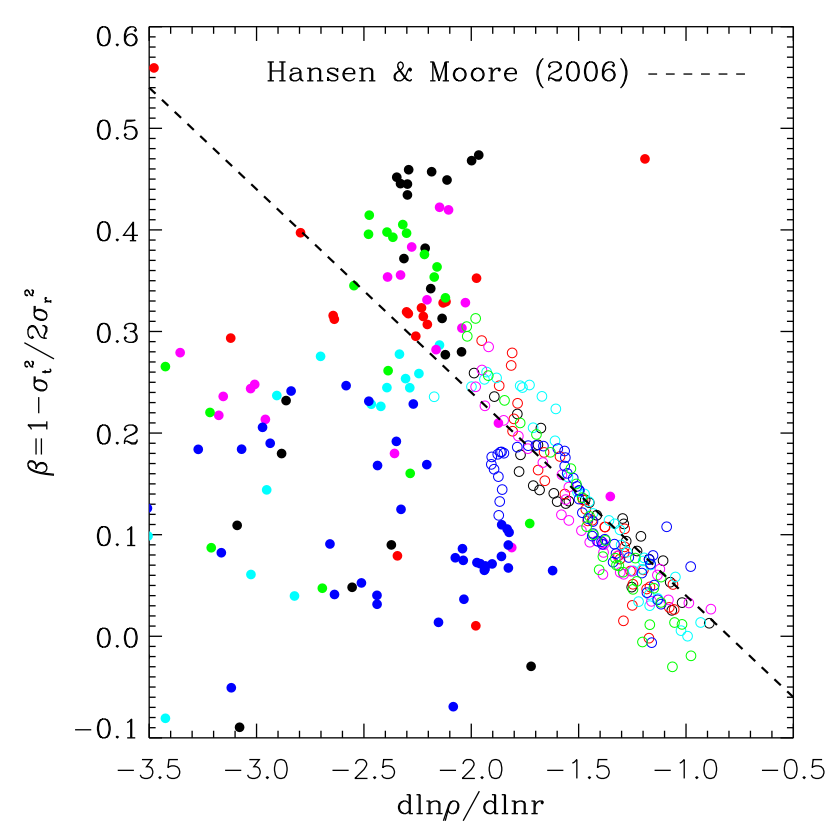

Figure 11. Local values of the logarithmic slope of the density profile plotted versus velocity anisotropy. The relation proposed by Hansen \& Moore (2006) is shown as a dashed line. Because the density profile steepens gradually from the centre outwards whereas the velocity anisotropy is non-monotonic, no simple relation between these two quantities is valid throughout the halos. The Hansen \& Moore formula approximates our results quite well in the inner regions, but large deviations may be seen outside $r_{-2}$, particularly at the largest radii where our halos are approximately isotropic but their density profiles are steepest. Open circles correspond to $r_{\mathrm{conv}}^{(1)}<r<r_{-2}$, filled circles to $r_{-2}<r<r_{200}$. Colors are as in Fig. 3.

\section{SUMMARY}

We have analysed density, velocity dispersion, anisotropy and pseudo-phase-space density profiles at redshift zero for simulated halos from the Aquarius Project. This is a set of six galaxy-sized halos whose formation and evolution have been simulated at a variety of resolutions in their proper $\Lambda \mathrm{CDM}$ context. The set includes the largest simulation of this kind reported so far; $\mathrm{a} \sim 4.4$ billion particle simulation in which the final halo has 1.1 billion particles within its virial radius, $r_{200}$. The set also includes simulations of all six halos with $100-200$ million particles within the virial radius, as well as a comprehensive numerical convergence study for the largest system. Our main conclusions are as follows.

- Density profiles deviate slightly but significantly from the NFW model, and are approximated well by a fitting formula where the logarithmic slope is a power-law of radius: the Einasto profile (eq. 4). The steeply-cusped profile of Moore et al. (1999) is a poor fit to the structure of our six halos.

- We find convincing evidence that the shape parameter of the Einasto formula varies from halo to halo at given mass (see Table 2). This complements the earlier conclusion of Merritt et al. (2006), Gao et al. (2008) and Hayashi \& 


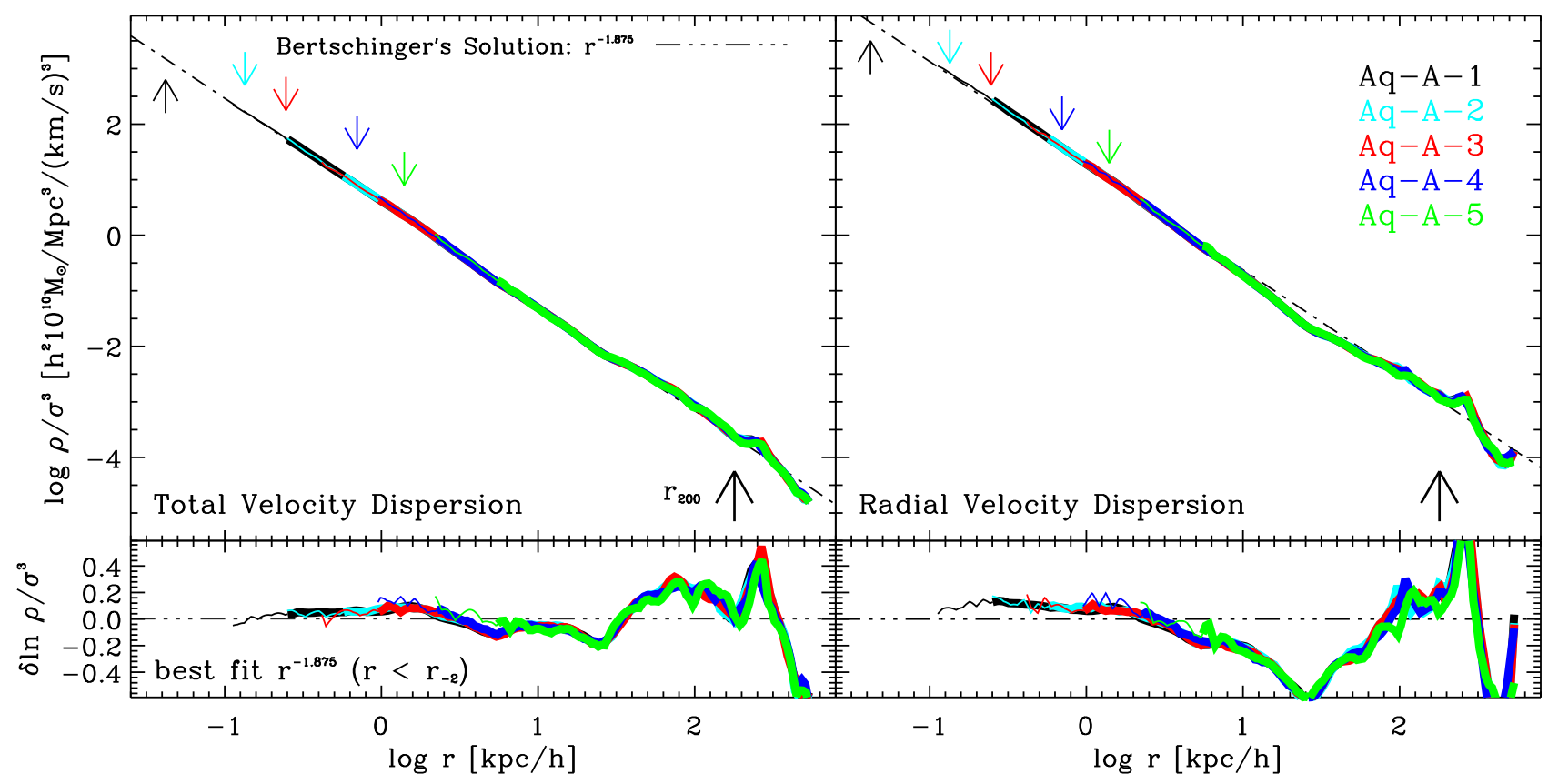

Figure 12. Pseudo-phase-space density profiles for our Aq-A convergence series, estimated as $\rho / \sigma^{3}$, computed in concentric spherical shells. Arrows, line-types, and colours are as in Fig. 1. Note the remarkable power-law behaviour of this quantity, a result already noted by Taylor \& Navarro (2001). The dot-dashed line is not a fit to the data, but rather the prediction of the similarity solution of Bertschinger (1985) for infall onto a point mass in an otherwise unperturbed Einstein-de Sitter universe, $\rho / \sigma^{3} \propto r^{-1.875}$. This has been scaled to match Aq-A at $r<r_{-2}$. Residuals from the Bertschinger solution are shown in the bottom panels. Note that this power-law behaviour is most evident when the full 3D velocity dispersion is used (left panels). When only the radial velocity dispersion is used (right panels) deviations from the Bertschinger solution are considerably larger.

White (2008) that its mean value varies systematically with halo mass. Together these results imply that the density profiles of $\Lambda \mathrm{CDM}$ halos are not strictly self-similar: different halos cannot be rescaled to look alike. This lack of similarity extends to the kinematic structure, as measured by the velocity dispersion and anisotropy profiles.

- Intriguingly, departures from similarity are minimized when analyzing a pseudo-phase-space density profile defined as $\rho / \sigma^{3}$. This suggests a limited sense in which $\Lambda \mathrm{CDM}$ halos are indeed nearly "universal". The pseudo-phase-space density profiles are very well approximated by $\rho / \sigma^{3} \propto r^{-1.875}$, the power law predicted by Bertschinger's similarity solution for infall onto a point mass in an otherwise unperturbed Einstein-de Sitter universe. This simple law has only one scaling parameter and no shape parameters, yet it approximates, for over six decades, the $\rho / \sigma^{3}$ profiles to better than $20-30 \%$, all the way from the innermost resolved point to the virial radius. The power-law description is, however, not perfect, and further work designed to understand better its origin and limitations seems warranted.

- Density profiles become monotonically shallower inwards, down to the innermost resolved point, with no indication that they approach power-law behaviour. The innermost slope we measure is slightly shallower than -1 , a result supported by estimates of the maximum possible asymptotic inner slope.

- These results convincingly rule out recent claims that typical $\Lambda \mathrm{CDM}$ halos may have asymptotic central cusps as steep as $r^{-1.2}$ (Diemand et al., 2004, 2005, 2008). Shallower cusps, such as the asymptotic $r^{-0.75}$ behaviour predicted by the model of Taylor \& Navarro (2001), cannot yet be excluded. These results should discourage further work assuming CDM cusps steeper than $r^{-1}$ except possibly around central black holes.

- Velocity anisotropy does not depend monotonically on radius beyond $r_{-2}$. Halos are roughly isotropic near the centre, are dominated by radial motions at intermediate radii, but become more isotropic again as the virial radius is approached. This behaviour does not appear to be driven by the presence of substructure. Given that the slope of the density profile does increase monotonically with radius, this implies that no simple relation between anisotropy and slope can hold throughout a halo. The relation recently proposed by Hansen \& Moore (2006) works reasonably well in the inner regions $\left(r<r_{-2}\right)$, but fails at larger radii.

The main aim of the Aquarius Project is to provide reliable theoretical predictions for the structure and formation history of dark matter halos like that surrounding the Milky Way down to radii of order $100 \mathrm{pc}$. This permits direct comparisons with a number of observations with minimal extrapolation, and it helps to design new observational strategies aimed at testing the cold dark matter paradigm on these very non-linear scales.

We recognize, however, that many of these tests and predictions will apply to regions where baryons play an important dynamical role. Our numerical work provides robust results for the limiting but unrealistic case of pure dark matter halos, and these will undoubtedly be modified in non- 


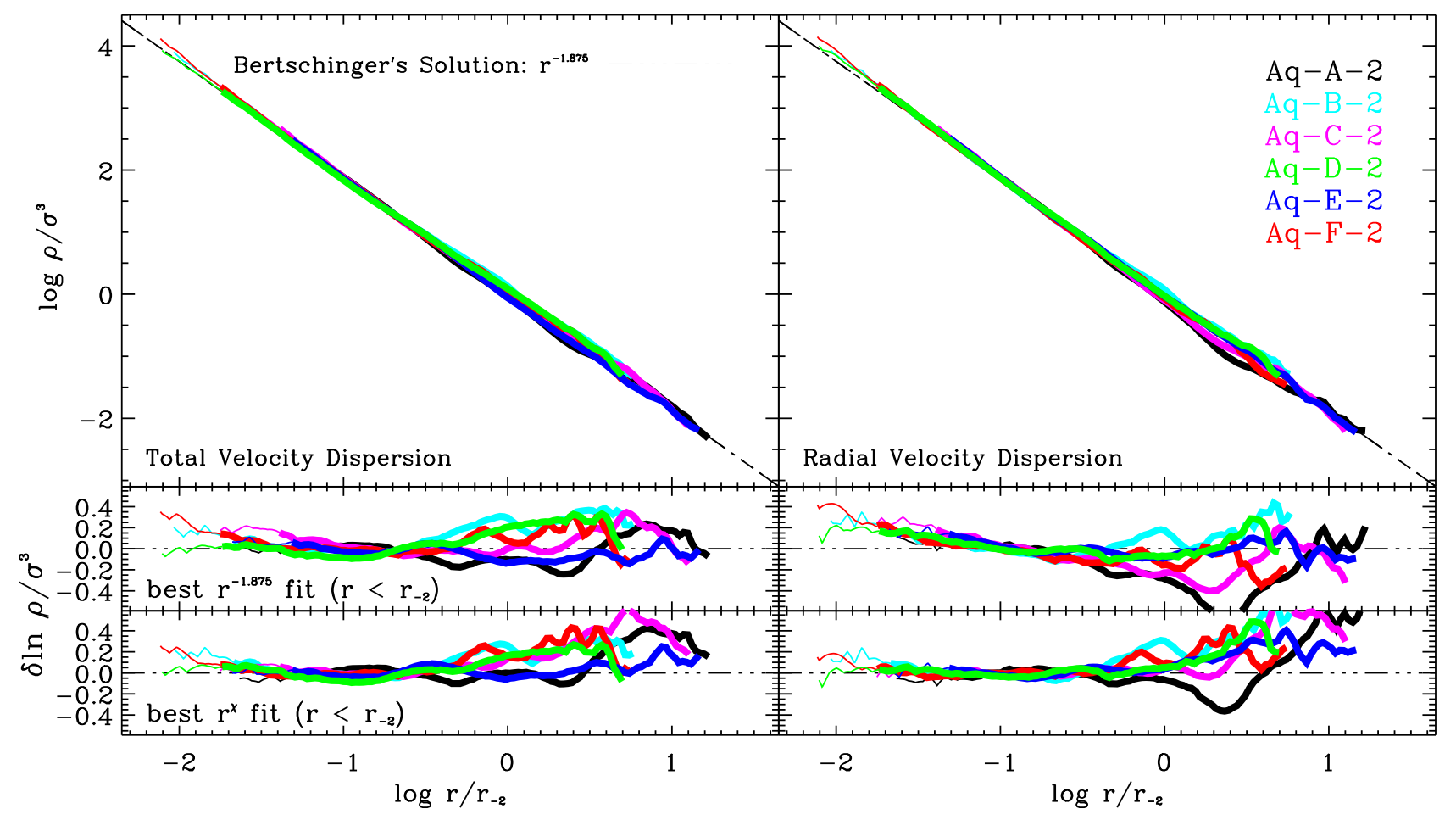

Figure 13. Pseudo-phase-space density profiles of all six level-2 Aquarius halos. Radii have been scaled to $r_{-2}$, and the pseudo-phasespace densities to maximise agreement within $r_{-2}$. Note that for all six halos these profiles are very well approximated by power laws with an exponent very close to that of the Bertschinger solution. All halos, including those that were outliers in the density, velocity dispersion, and anisotropy profiles, are almost indistinguishable in this plot. Deviations from the Bertschinger law are typically more pronounced when radial velocity dispersion is used instead of the full 3D velocity dispersion. Residuals from the best-fit power-laws, $\rho / \sigma^{3} \propto r^{\chi}$, are shown in the bottom panels. The values of $\chi$ are listed for each halo in Table 2 .

trivial ways by the presence of baryons. Providing a full account of the coupled structure of the cold dark matter and baryonic components in galaxies like our own is clearly the next major computational challenge, and it is likely to exercise us for some time to come.

\section{ACKNOWLEDGMENTS}

The simulations for the Aquarius Project were carried out at the Leibniz Computing Center, Garching, Germany, at the Computing Centre of the Max-Planck-Society in Garching, at the Institute for Computational Cosmology in Durham, and on the 'STELLA' supercomputer of the LOFAR experiment at the University of Groningen. This work was supported in part by an STFC rolling grant to the ICC. CSF acknowledges a Royal Society Wolfson Research Merit award. AH acknowledges financial support from NOVA and NWO.

\section{REFERENCES}

Allgood B., Flores R. A., Primack J. R., et al., 2006, MNRAS, 367,1781

Bertschinger E., 1985, ApJS, 58, 39

Dehnen W., McLaughlin D. E., 2005, MNRAS, 363, 1057

Diemand J., Kuhlen M., Madau P., 2007, ApJ, 657, 262
Diemand J., Kuhlen M., Madau P., et al., 2008, Nature, 454, 735 Diemand J., Moore B., Stadel J., 2004, MNRAS, 353, 624

Diemand J., Zemp M., Moore B., Stadel J., Carollo C. M., 2005, MNRAS, 364, 665

Einasto J., 1965, Trudy Inst. Astroz. Alma-Ata, 51, 87

Eke V. R., Cole S., Frenk C. S., 1996, MNRAS, 282, 263

Frenk C. S., White S. D. M., Davis M., Efstathiou G., 1988, ApJ, 327,507

Fukushige T., Makino J., 2001, ApJ, 557, 533

Gao L., Navarro J. F., Cole S., et al., 2008, MNRAS, 387, 536

Gao L., White S. D. M., Jenkins A., Stoehr F., Springel V., 2004, MNRAS, 355, 819

Ghigna S., Moore B., Governato F., Lake G., Quinn T., Stadel J., 2000, ApJ, 544, 616

Graham A. W., Merritt D., Moore B., Diemand J., Terzic B., 2006, AJ, 132, 2701

Hansen S. H., Moore B., 2006, New Astronomy, 11, 333

Hayashi E., Navarro J. F., Springel V., 2007, MNRAS, 377, 50

Hayashi E., White S. D. M., 2008, MNRAS, 388, 2

Henry J. P., Evrard A. E., Hoekstra H., Babul A., Mahdavi A., 2008, ArXiv e-prints

Jing Y. P., Suto Y., 2002, ApJ, 574, 538

Klypin A., Kravtsov A. V., Valenzuela O., Prada F., 1999, ApJ, 522,82

Komatsu E., Dunkley J., Nolta M. R., et al., 2008, ArXiv e-prints, 0803.0547

Kuhlen M., Diemand J., Madau P., 2008, ArXiv e-prints, 0805.4416

Merritt D., Graham A. W., Moore B., Diemand J., Terzić B., 


\begin{tabular}{llllllllll}
\hline \hline Halo & $\begin{array}{l}m_{\mathrm{p}} \\
{\left[M_{\odot} / h\right]}\end{array}$ & $\begin{array}{l}\epsilon_{G} \\
{[\mathrm{pc} / h]}\end{array}$ & $\begin{array}{l}r_{200} \\
{[\mathrm{kpc} / h]}\end{array}$ & $\begin{array}{l}M_{200} \\
{\left[M_{\odot} / h\right]}\end{array}$ & $\begin{array}{l}N_{200} \\
{\left[10^{6}\right]}\end{array}$ & $\begin{array}{l}V_{\max } \\
{[\mathrm{km} / \mathrm{s}]}\end{array}$ & $\begin{array}{l}r_{\max } \\
{[\mathrm{kpc} / h]}\end{array}$ & $\begin{array}{l}\sigma_{\text {host }} \\
{[\mathrm{km} / \mathrm{s}]}\end{array}$ & $\begin{array}{l}\sigma_{\max } \\
{[\mathrm{km} / \mathrm{s}]}\end{array}$ \\
\hline Aq-A-1 & $1.250 \times 10^{3}$ & 14 & 179.41 & $1.343 \times 10^{12}$ & 1074.06 & 208.75 & 20.69 & 117.47 & 261.70 \\
Aq-A-2 & $1.000 \times 10^{4}$ & 48 & 179.49 & $1.345 \times 10^{12}$ & 134.47 & 208.49 & 20.54 & 117.13 & 261.88 \\
Aq-A-3 & $3.585 \times 10^{4}$ & 87 & 179.31 & $1.341 \times 10^{12}$ & 37.39 & 209.22 & 20.35 & 117.31 & 262.80 \\
Aq-A-4 & $2.868 \times 10^{5}$ & 250 & 179.36 & $1.342 \times 10^{12}$ & 4.68 & 209.24 & 20.58 & 117.23 & 262.29 \\
Aq-A-5 & $2.294 \times 10^{6}$ & 500 & 180.05 & $1.357 \times 10^{12}$ & 0.59 & 209.17 & 20.84 & 116.61 & 260.59 \\
\hline Aq-B-2 & $4.706 \times 10^{3}$ & 48 & 137.02 & $5.982 \times 10^{11}$ & 127.09 & 157.68 & 29.31 & 89.59 & 190.74 \\
Aq-C-2 & $1.021 \times 10^{4}$ & 48 & 177.26 & $1.295 \times 10^{12}$ & 126.77 & 222.40 & 23.70 & 124.08 & 270.50 \\
Aq-D-2 & $1.020 \times 10^{4}$ & 48 & 177.28 & $1.295 \times 10^{12}$ & 126.98 & 203.20 & 39.48 & 113.15 & 254.28 \\
Aq-E-2 & $7.002 \times 10^{3}$ & 48 & 154.96 & $8.652 \times 10^{11}$ & 123.56 & 179.00 & 40.52 & 101.73 & 215.14 \\
Aq-F-2 & $4.946 \times 10^{3}$ & 48 & 152.72 & $8.282 \times 10^{11}$ & 167.45 & 169.08 & 31.15 & 96.78 & 204.53 \\
\hline
\end{tabular}

Table 1. Basic parameters of the Aquarius simulations. We have simulated 6 different halos, each at several different numerical resolutions. The leftmost column gives the simulation name, encoding the halo (A to F), and the resolution level ( 1 to $5 ; 1$ is our highest resolution, 5 the lowest). $m_{\mathrm{p}}$ is the particle mass in the high-resolution region, $\epsilon_{G}$ is the Plummer-equivalent gravitational softening length, $r_{200}$ is the virial radius, defined as the radius enclosing a mean overdensity 200 times the critical value for closure, $M_{200}$ is the mass within the virial radius, $N_{200}$ is the total number of particles within $r_{200}$. Other characteristic properties of the halos listed are the position $\left(r_{\text {max }}\right)$ of the peak $\left(V_{\max }\right)$ of the circular velocity profile, as well as the $1 \mathrm{D}$ velocity dispersion of the main halo $\left(\sigma_{\text {host }}\right)$, and the peak $\left(\sigma_{\text {max }}\right)$ of the velocity dispersion profile.

\begin{tabular}{lllllllll}
\hline \hline Halo & $\begin{array}{l}r_{\text {conv }}^{(1)} \\
{[\mathrm{kpc} / h]}\end{array}$ & $\begin{array}{l}r_{\text {conv }}^{(7)} \\
{[\mathrm{kpc} / h]}\end{array}$ & $\begin{array}{l}\rho_{-2} \\
{\left[10^{10} h^{2} M_{\odot} / \mathrm{Mpc}^{3}\right]}\end{array}$ & $\begin{array}{l}r_{-2} \\
{[\mathrm{kpc} / h]}\end{array}$ & $\alpha$ & $\chi$ & $\chi_{r}$ & $\gamma_{\max }$ \\
\hline Aq-A-1 & 0.113 & 0.253 & $7.462 \times 10^{5}$ & 11.05 & $0.170 \pm 0.0259$ & -1.898 & -1.948 & 0.894 \\
Aq-A-2 & 0.250 & 0.575 & $7.322 \times 10^{5}$ & 11.15 & $0.163 \pm 0.0249$ & -1.917 & -1.976 & 1.051 \\
Aq-A-3 & 0.417 & 0.966 & $7.456 \times 10^{5}$ & 11.09 & $0.174 \pm 0.0266$ & -1.926 & -1.995 & 1.128 \\
Aq-A-4 & 0.952 & 2.277 & $6.501 \times 10^{5}$ & 11.90 & $0.160 \pm 0.0248$ & -1.991 & -2.061 & 1.321 \\
Aq-A-5 & 2.206 & 5.530 & $7.534 \times 10^{5}$ & 11.02 & $0.165 \pm 0.0268$ & -2.015 & -2.111 & 1.493 \\
\hline Aq-B-2 & 0.219 & 0.507 & $1.830 \times 10^{5}$ & 16.79 & $0.173 \pm 0.0123$ & -1.868 & -1.938 & 1.039 \\
Aq-C-2 & 0.248 & 0.573 & $4.973 \times 10^{5}$ & 14.37 & $0.159 \pm 0.0125$ & -1.948 & -2.010 & 1.077 \\
Aq-D-2 & 0.281 & 0.652 & $2.075 \times 10^{5}$ & 20.30 & $0.170 \pm 0.0124$ & -1.862 & -1.942 & 1.070 \\
Aq-E-2 & 0.223 & 0.516 & $2.058 \times 10^{5}$ & 17.88 & $0.130 \pm 0.0200$ & -1.912 & -1.947 & 1.084 \\
Aq-F-2 & 0.209 & 0.486 & $1.673 \times 10^{5}$ & 18.84 & $0.145 \pm 0.0167$ & -1.911 & -1.980 & 1.298 \\
\hline
\end{tabular}

Table 2. Fit parameters of Aquarius halos. The first column labels each halo, as in Table 1, the second and third list the convergence radii obtained for $\kappa=1$ and $\kappa=7$. These radii, $r_{\mathrm{conv}}^{(1)}$ and $r_{\mathrm{conv}}^{(7)}$, respectively, correspond to where departures from convergence in the circular velocity are expected to be of order $10 \%$ and $2.5 \%$. The characteristic scale radius $r_{-2}$ corresponds to where the logarithmic slope equals the isothermal value; $\rho_{-2}=\rho\left(r_{-2}\right)$, and $\alpha$ is the best-fit Einasto parameter. The uncertainty in $\alpha$ indicates the range where $\Delta Q / Q$ deviates by less than $50 \%$ from the absolute minimum shown in Fig. 4 . Strictly, these are non-symmetric, so we conservatively quote the largest deviation, positive or negative. $\chi$ refers to the exponent of the best fitting power-law describing the $\rho / \sigma^{3}$ profile. $\chi_{r}$ is analogous to $\chi$, but for $\rho / \sigma_{r}^{3}$, where $\sigma_{r}$ is the rms velocity in radial motions. $\chi$ and $\chi_{r}$ are computed by minimizing residuals in the region $r_{\text {conv }}^{(1)}<r<r_{-2}$. Finally, $\gamma_{\max }$ lists the value of the maximum asymptotic slope of the density profile cusp, measured at $r=r_{\mathrm{conv}}^{(7)}$.

2006, AJ, 132, 2685

Merritt D., Navarro J. F., Ludlow A., Jenkins A., 2005, ApJL, $624, \mathrm{~L} 85$

Moore B., Ghigna S., Governato F., et al., 1999a, ApJL, 524, L19

Moore B., Quinn T., Governato F., Stadel J., Lake G., 1999b, MNRAS, 310, 1147

Navarro J. F., Frenk C. S., White S. D. M., 1996, ApJ, 462, 563 Navarro J. F., Frenk C. S., White S. D. M., 1997, ApJ, 490, 493

Navarro J. F., Hayashi E., Power C., et al., 2004, MNRAS, 349, 1039

Power C., Navarro J. F., Jenkins A., et al., 2003, MNRAS, 338, 14

Prugniel P., Simien F., 1997, A\&A, 321, 111

Sersic J. L., 1968, Atlas de galaxias australes, Cordoba, Argentina: Observatorio Astronomico, 1968

Spergel D. N., Verde L., Peiris H. V., et al., 2003, ApJS, 148, 175
Springel V., 2005, MNRAS, 364, 1105

Springel V., White S. D. M., Jenkins A., et al., 2005, Nature, 435 , 629

Springel V., Yoshida N., White S. D. M., 2001, New Astronomy, 6,79

Springel et al., 2008a, ArXiv e-prints, 0809.0894

Springel et al., 2008b, ArXiv e-prints, 0809.0898

Stadel J., Potter D., Moore B., et al., 2008, ArXiv e-prints, 808

Stoehr F., 2006, MNRAS, 365, 147

Stoehr F., White S. D. M., Springel V., Tormen G., Yoshida N., 2003, MNRAS, 345, 1313

Taylor J. E., Navarro J. F., 2001, ApJ, 563, 483

Vass I., Valluri M., Kravtsov A., Kazantzidis S., 2008, ArXiv eprints

Vogelsberger M., Helmi A., Springel V., et al., 2009, MNRAS, 395,797 
White S. D. M., 1996, in Cosmology and Large-Scale Structure, edited by R. Schaefer, J. Silk, M. Spiro, J. Zinn-Justin, Dordrecht: Elsevier, astro-ph/9410043 